Check for updates

Cite this: J. Mater. Chem. A, 2018, 6, 16292

Received 6th April 2018

Accepted 8th August 2018

DOI: $10.1039 / c 8 t a 03159 b$

rsc.li/materials-a

\section{Metal-organic framework nanosheets (MONs): a new dimension in materials chemistry}

\begin{abstract}
David J. Ashworth (D) and Jonathan A. Foster (D)*
Metal-organic framework nanosheets (MONs) are emerging as a novel class of two-dimensional materials with a distinct set of design principles, synthetic approaches, characterisation techniques and applications. MONs are free standing, nominally two-dimensional materials formed by the co-ordination of organic ligands to metal ions or clusters. In comparison to other metal-organic and two-dimensional materials, the principles behind their design and synthesis are only just beginning to be understood. Here we seek to bring together recent highlights from this rapidly growing field and attempt to draw out common principles and strategies which we hope will aid the development of this exciting new class of materials. We consider the range of chemistries and different synthetic strategies used to fabricate MONs, the methods employed to characterise them and the applications that have so far been investigated.
\end{abstract}

\section{Introduction}

Two-dimensional materials offer a distinct set of mechanical, electronic, optical and magnetic properties compared to their bulk, layered forms. Graphene is the archetypal twodimensional material and consists of a single layer of $\mathrm{sp}^{2}$ hybridised carbon atoms giving it exceptional conductivity,

Department of Chemistry, The University of Sheffield, Dainton Building, Sheffield, S3 7HF, UK. E-mail: jona.foster@sheffield.ac.uk tensile strength, flexibility and transparency. ${ }^{1} \mathrm{~A}$ wide variety of other elemental and inorganic two-dimensional materials with complimentary properties have also been investigated including boron nitride, ${ }^{2}$ black phosphorus, ${ }^{3}$ metal-oxides, ${ }^{4}$ double layer metal hydroxides, ${ }^{5}$ transition metal dichalcogenides, ${ }^{6}$ clays and zeolites. ${ }^{7}$ However, despite the remarkable properties shown by many of these materials, their simple composition means that it is often challenging to modify or systematically tune their structures in order to optimise these properties for particular applications.

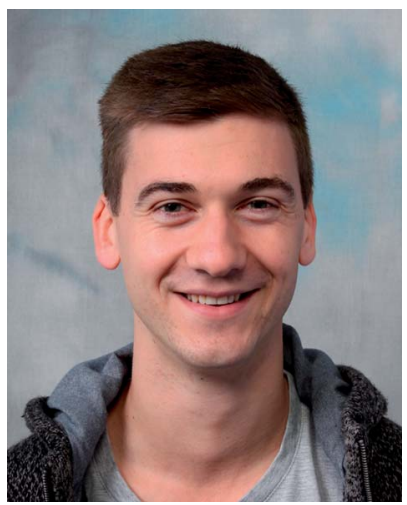

Dave Ashworth was awarded his MChem, with study in industry, degree in 2016 from the University of Sheffield, under the tutorship of Prof. Lee Brammer and Dr Jonathan Foster. He is currently studying for his PhD at the same institution, under the supervision of Dr Foster. His research centres around the development and chemical understanding of novel twodimensional metal-organic framework nanosheets and their potential applications.

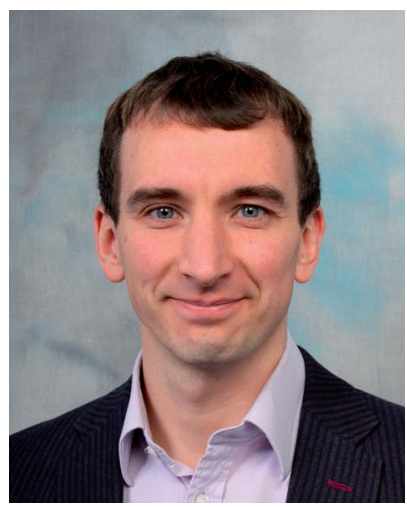

Dr Jonathan Foster graduated with an MChem from the University of Durham in 2008 where he also completed a PhD in the groups of Prof. Jonathan Steed and Prof. Judith Howard CBE FRS. He undertook postdoctoral positions at the University of Cambridge in the groups of Prof. Jonathan Nitschke and Prof. Anthony Cheetham FRS. In 2015 he was awarded a Ramsay Memorial Trust Fellowship and the position of Vice Chancellors Fellow which he holds in the department of Chemistry at the University of Sheffield. Jonathan has experience working with a wide range of nano-structured supramolecular materials including gels, polymers, cages and frameworks. His current research is focussed on realising the potential of metal-organic framework nanosheets for use in creating novel sensors, catalysts, composite materials and devices. 
Metal-organic materials combine the diversity of organic ligands with the unique properties of metal ions to produce robust materials with well-defined and readily tuneable structures. This approach has been used to create a variety of different metal-organic architectures ranging from discrete complexes, cages, grids, helicates and knots to extended polymers, gels, liquids and liquid crystals. ${ }^{8-12}$ In particular, there are over 70000 thousand metal-organic frameworks (MOFs) reported in the Cambridge structural database (CSD), many of which have a layered structure. ${ }^{13}$ A number of well-established coordination motifs exist allowing for a high degree of predictability over the topology of the structures that will form. The modular nature of these materials often allows them to be systematically tuned and modified through reticular substitution of different ligands and metal ions and through postsynthetic modifications. The versatility of metal-organic materials means they have been exploited for applications as diverse as gas-storage,${ }^{14}$ water purification, ${ }^{15}$ catalysis,${ }^{16}$ drug-delivery, ${ }^{17}$ sensing, ${ }^{18}$ imaging $^{19}$ and electronics. ${ }^{20}$

Two-dimensional metal-organic framework nanosheets (MONs) have emerged as the most recent form-factor for metalorganic materials. MONs have been variously described in the literature as metal-organic framework nanosheets, ${ }^{21}$ twodimensional MOFs, ${ }^{22}$ metal-organic layers (MOLs), ${ }^{23}$ metalorganic graphene analogues (MOGs), ${ }^{24}$ metal-organic surfaces, ${ }^{25}$ single-layered MOF based materials (MOFenes), ${ }^{26}$ metal-organic flakes,${ }^{27}$ two-dimensional coordination polymers ${ }^{28}$ coordination nanosheets (CONASH), ${ }^{29}$ organometallic sheets, ${ }^{30,31}$ and hybrid organic-inorganic nanosheets. ${ }^{32}$ Whilst there are meaningful distinctions between some of the terms used in these descriptions, in an effort to draw out the commonality in the underlying chemistry, approaches to synthesis, techniques for characterisation and applications envisaged we group them here under a common name.

The term "nanosheet" has been used extensively in the MOF literature and whilst a large number of layered MOFs exist or have been grown on surfaces, in most cases the individual layers will not be stable or cannot be isolated using a given method. However, as with other two-dimensional materials, the ideals of a uniform crystalline material with single unit thickness extended infinitely in the other two dimensions is not often achieved in practice. Here we employ a broad definition which encompasses materials which clearly display 'nanosheet'-like structure and properties and that, with iterative development, could form free standing single-layers. More specifically, MONs consist of: [1] organic ligands coordinated to metal ions or clusters with continuous connectivity in two-dimensions but only non-covalent interactions in the third dimension; [2] highly anisotropic materials with one dimension approaching monolayer thickness and the others being at least an order of magnitude larger and approximately equal in size; [3] materials which can be isolated in a form with the dimensions outlined above as free standing sheets, not attached to a surface or other scaffold or as layers in a bulk material.

In this critical review we shall describe recent progress in this rapidly developing area and seek to highlight the breadth of approaches taken and draw out informative examples of good practice and recent innovations. We begin in Section 2 by discussing the diverse range of chemistries used to synthesise MONs and attempt to draw-out common design principles. In Section 3 we present different approaches for synthesising MONs, either directly or from their parent layered frameworks. We will then discuss the range of characterisation techniques that have developed for analysing the molecular, nanoscopic and macroscopic structure and properties of MONs in Section 4. In Section 5 we consider the distinct opportunities offered by MONs for use in a wide range of applications before summarising and providing our own perspective on the future of this field in Section 6.

\section{The anatomy of MONs}

The basic principle behind the structure of two-dimensional nanosheets is to design materials with strong, directional interactions within a plane, but weak interactions between the layers in the bulk material. This is exemplified by graphene in which strong covalent carbon-carbon bonds form a hexagonal lattice, layers of which are held together by relatively weak dispersion interactions. ${ }^{33}$ Connectivity within the layers of MONs can be achieved either through organic ligands which bridge isolated metal ions or clusters or through connected inorganic clusters, or a mixture of the two. ${ }^{34}$ Any connectivity in the third dimension is typically a mixture of dispersive, hydrogen bonding and ionic interactions which allows for the individual layers to be separated out and isolated. Here we seek to capture the diversity of structures investigated so far by bringing together examples of MONs which share common ligands and structural motifs.

\subsection{Carboxylate based MONs}

As with MOFs, the most popular organic ligands for the synthesis of MONs are polycarboxylates thanks to their strong, directional coordination chemistry and the wide variety of ligands commercially available. The paddlewheel (PW) motif is an ideal secondary building unit (SBU) for creating MONs as it consists of four carboxylate ligands organised in a plane around two metal cations capped with axial ligands (Fig. 1a). The archetypal MOF-2 was amongst the first layered MOFs to be exfoliated to form MONs and consists of layers of 1,4-benzene dicarboxylate (BDC) coordinated via $\mathrm{Zn}$ paddlewheels with water molecules occupying the axial positions of the paddlewheel. ${ }^{35}$ Isostructural MONs have since been formed using a variety of metal ions (Cu, $\mathrm{Zn}, \mathrm{Co})$ and 1,4-BDC ${ }^{36-38}$ and 1,3-BDC derivatives, ${ }^{39-41}$ as well as longer linear diacid chains. ${ }^{42}$ In our own work, we functionalised 1,4-BDC with weakly interacting alkylether chains designed to weaken interlayer interactions and aid exfoliation into solvent (Fig. 1c). ${ }^{43}$ Several MONs based on tetrakis(4-carboxy-phenyl)porphyrin (TCPP) structure have been synthesised (Fig. 1d) in combination with a variety of metal-PW's (Zn, Cu, Cd, Co). ${ }^{44-55}$ This motif also allows for the formation of bimetallic MONs by using different metal ions coordinated at the PW and porphyrin sites. ${ }^{45,52}$ The high degree of connectivity and preorganisation afforded by the porphyrin units makes them particularly well suited to the formation of MONs. 
a)

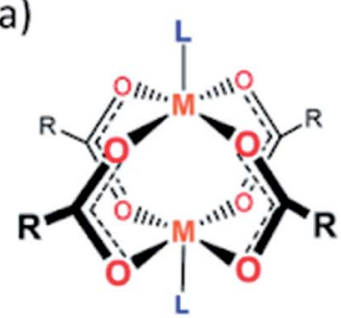

e)

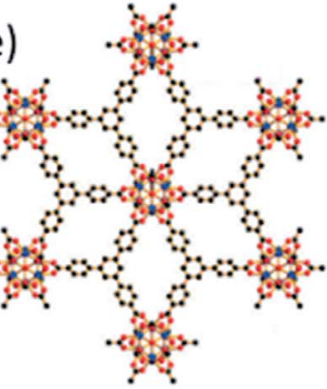

b)

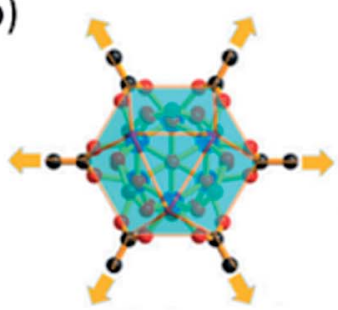

f)

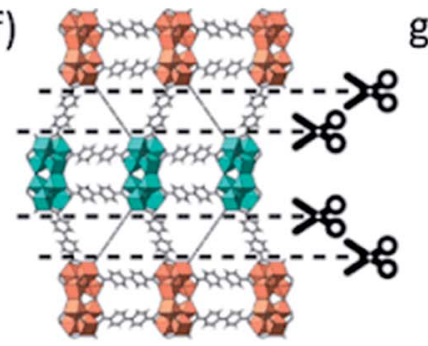

c)

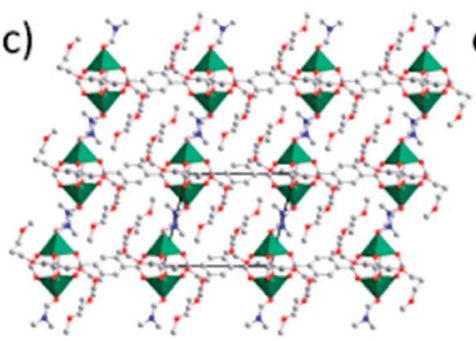

d)

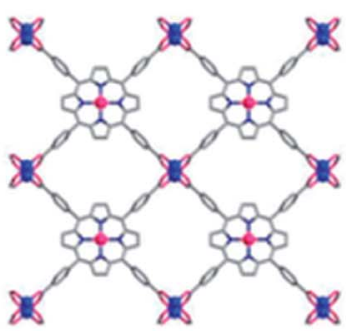

Fig. 1 Schemes showing (a) paddlewheel and (b) $M_{6} L_{6}$ secondary building units. (c $-\mathrm{h}$ ) example crystal structures showing diversity of carboxylic acid based linkers and secondary building units used in the synthesis of MONs. Specifically, (c) $\mathrm{Zn}_{2}\left(\mathrm{BDC}-\mathrm{x}_{2}\right)(\mathrm{DMF})_{2}, w h e r e \mathrm{x}=\mathrm{O}\left(\mathrm{CH} \mathrm{H}_{3} \mathrm{OMe}\right.$; $(\mathrm{d})$ $\mathrm{M}(\mathrm{TCPP})$ where $\mathrm{M}=\mathrm{Zn}, \mathrm{Cu}, \mathrm{Cd}, \mathrm{Co}$; (e) $\left[\mathrm{Hf}_{6}\left(\mu_{3}-\mathrm{O}\right)_{4}\left(\mu_{3}-\mathrm{OH}\right)_{4}(\text { carboxylate })_{12}\right]$; (f) hcp UiO-67; (g) Mn(DMS)( $\left.\mathrm{H}_{2} \mathrm{O}\right)$; (h) lanthanum 1,3,5-benzenetriphosphonate (LBP-II). Adapted with permission from ref. 56, 43, 52, 56, 60, 32 and 65 for images (b-h) respectively.

$\mathrm{Zr}$ and Hf carboxylate clusters have proved popular SBU's for creating robust 3D MOFs but have only recently be used to create 2D MONs. Cao et al. synthesised a series of MONs by combining 3-connected carboxylate ligands, initially benzene1,3,5-tribenzoate (BTB) moieties, with the $\mathrm{Hf}^{4+}$ cluster $\left[\mathrm{Hf}_{6}\left(\mu_{3^{-}}\right.\right.$ O) $\left.{ }_{4}\left(\mu_{3}-\mathrm{OH}\right)_{4}(\text { carboxylate })_{12}\right]$ to create an infinite 3,6-connected 2D network with kagome dual (kgd) topology (Fig. 1e). ${ }^{56}$ The 12connectivity of the $\mathrm{Hf}_{6}$ cluster violates the geometric requirement of a 2D layer so they used formate to cap six of the connection sites on the cluster, leaving the remaining six in the same plane to connect to the BTB ligands (Fig. 1b). Zhao and coworkers produced analogous layered structures based on BTB with both $\mathrm{Hf}$ and $\mathrm{Zr} \mathrm{M} \mathrm{M}_{6} \mathrm{O}_{4}(\mathrm{OH})_{4}$ clusters synthesised using a modulated hydrothermal approach ${ }^{57}$ and Wang et al. recently demonstrated a continuous flow reaction in order to synthesise the $\mathrm{Zr}$ analogue. ${ }^{58}$ The same $\mathrm{Zr}_{6}$ cluster but with different sites capped was used to create MONs with tetraphenylethylenebased tetracarboxylate (TCBPE), ${ }^{23}$ as well as TCPP ligands. ${ }^{59} \mathrm{~A}$ $\mathrm{Hf}_{12}$ cluster was used by Cliffe $e t$ al. who used 4,4-biphenyldicarboxylate $\left(\mathrm{bpdc}^{2-}\right)$ to form a 3D framework containing a "double cluster" $\left(\mathrm{Hf}_{12} \mathrm{O}_{8}(\mathrm{OH})_{14}\right)$ which they were able to exfoliate through selective scission of the interlayer ligands (Fig. 1f) ${ }^{60} \mathrm{~A}$ related double-decker $\mathrm{Hf}_{12}$ clusters has also been used as an SBU with extended tritopic carboxylates. ${ }^{61}$

Inorganic (I) corner sharing octahedra have also been used alongside coordination bonds (O) to create two-dimensional layers. In the nomenclature defined by Cheetham, Rao and Fuller, superscript numbers are used to define the types of connectivity in different dimensions. ${ }^{34}$ Cheetham and coworkers combined 2,2-dimethylsuccinate (DMS) and its isomers with different metal ions to produce a series of layered frameworks which they exfoliated to form nanosheets. ${ }^{32,62,63}$ Fig. $1 \mathrm{~g}$ shows the archetypal $\mathrm{I}^{1} \mathrm{O}^{1}$ network for the Mn-DMS network in which distorted $\mathrm{MnO}_{6}$ octahedra form corner-sharing chains along the $b$-axis which are bridged via DMS ligands to form 2D layers. ${ }^{32,64}$ The methyl groups of the ligands protrude between the layers providing weakly interacting hydrophobic caps. Other closely related systems had either inorganic $\left(\mathrm{I}^{2} \mathrm{O}^{0}\right)$ or organic connectivity in two dimensions $\left(\mathrm{I}^{0} \mathrm{O}^{2}\right)$ highlighting the challenges associated with predicting structure when using less preorganised ligands. Phosphonic acids can also be used to create inorganic layers. Araki et al. exploit the high coordination numbers of lanthanide ions by coordinating 1,3,5-benzenetriphosphonate to lanthanum ions to form layered MOFs with either $\left(\mathrm{I}^{1} \mathrm{O}^{1}\right)$ or $\left(\mathrm{I}^{2} \mathrm{O}^{0}\right)$ connectivity (Fig. $\left.1 \mathrm{~h}\right){ }^{65}$

\subsection{N-Donor based MONs}

Zeolitic imidazolate frameworks (ZIFs) are a highly successful class of MOF due to their relatively high stability, diversity and structural similarity to zeolites. ${ }^{66}$ However, the topology of imidazole-zinc coordination does not automatically lend itself to the formation of layered structures. Lotsch and coworkers used the lamellar surfactant CTAB to template benzimidazole (bim) and $\mathrm{Zn}$ to form a ZIF with the composition $\mathrm{Zn}(\mathrm{bi}$ $\mathrm{m})(\mathrm{OAc}){ }^{67}$ The layers are composed of $\mathrm{Zn}(\mathrm{bim})_{2 / 2}$ chains and $\mathrm{Zn}(\mathrm{OAc})_{2 / 2}$ chains connected by $\mathrm{Zn}^{2+}$ tetrahedra which stack together through weak van der Waals interactions (Fig. 2a). Peng et al. successfully exfoliated large nanosheets from a different layered $\mathrm{ZIF}$ with the composition $\mathrm{Zn}_{2}(\mathrm{bim})_{4}$. Here the layers are formed by $\mathrm{Zn}$ atoms coordinated to four bim ligands in a distorted tetrahedral geometry, and each bim ligand bridges two $\mathrm{Zn}$ atoms via a bis-monodentate linkage (Fig. 2b). ${ }^{68,69}$ It should also be noted that the reaction of 2methylimidazole (Hmim) with zinc nitrate in water at room temperature produces highly anisotropic leaf shaped crystals of 
a)
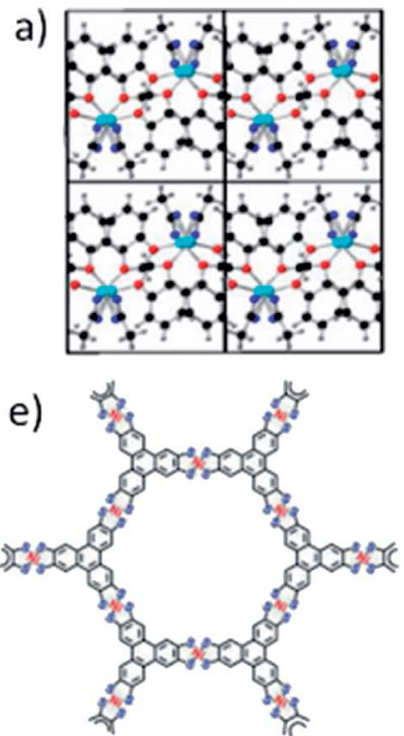

b)

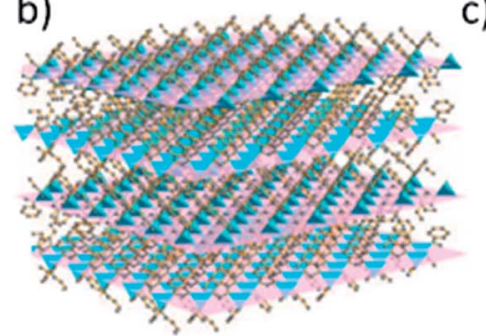

f)

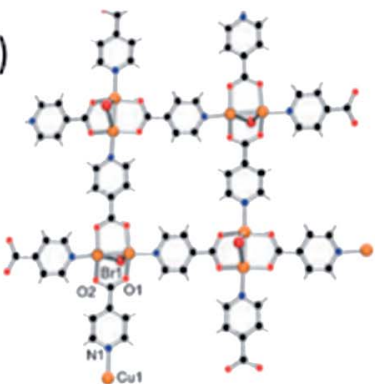

c)

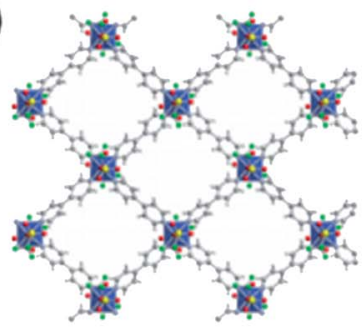

d)

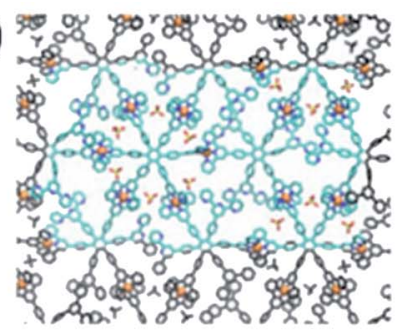

g)

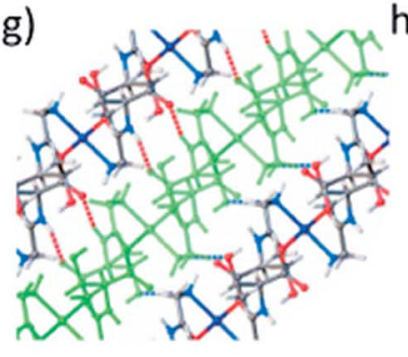

h)

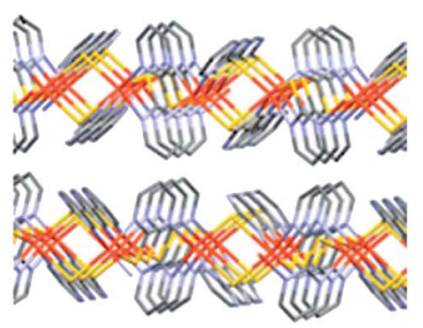

Fig. 2 Example structures of nitrogen $(a-d)$, sulfur (e) and mixed linkers ( $f-h)$ used in the synthesis of MONs. Specific repeat structural units are: (a) $\mathrm{Zn}\left(\right.$ bim) $(\mathrm{OAc})$; (b) $\mathrm{Zn}_{2}(\text { (bim) })_{4}$; (c) $\mathrm{Cu}(\mathrm{bpy})_{2}(\mathrm{OTf})_{2}$, (d) $\mathrm{S} 1\left(\mathrm{M}^{2+}\right)$, where $\mathrm{M}=\mathrm{Zn}, \mathrm{Fe}, \mathrm{Co}, \mathrm{Pd}$ and S1-see reference; (e) $\mathrm{Ni}_{3}(\mathrm{bis}(\mathrm{dithiolene}))_{2}$; (f) $\left[\mathrm{Cu}_{2} \mathrm{Br}(\mathrm{IN})_{2}\right]$ where IN = isonicotinate; (g) $\left[\mathrm{Zn}(\mathrm{Gly}-\mathrm{Thr})_{2}\right] \cdot \mathrm{CH}_{3} \mathrm{OH}$; (h) $\left[\mathrm{Fe}\left(\mathrm{Py}_{2} \text { th) }\right)_{2}\right]$. Adapted with permission from ref. 67, 68, 74, 76, 80-83 for images $(a-h)$ respectively.

ZIF-L $\left(\mathrm{Zn}(\mathrm{mim})_{2} \cdot(\mathrm{Hmim})_{1 / 2} \cdot\left(\mathrm{H}_{2} \mathrm{O}\right)_{3 / 2}\right)$ with thicknesses as low as $\sim 100 \mathrm{~nm}$. Several studies exploiting the sheet like properties of these materials for gas separation, ${ }^{70}$ templation $^{71}$ and catalysis ${ }^{72}$ have been reported, although sheets approaching monolayer thickness are, to our knowledge, yet to be reported. However, nanosheets of the closely related ZIF-67, $\mathrm{Co}(\mathrm{mim})_{2}$, formed using a salt templated synthesis have recently been reported. ${ }^{73}$

The neutral N-donor systems, 4,4-bipyridine (bpy), was used to create a layered framework with the formula $\mathrm{Cu}(\mathrm{bpy})_{2}(\mathrm{OTf})_{2}$ in which the trifluoromethanesulfonate (OTf) counterions capped weakly interacting layers (Fig. 2c). ${ }^{74}$ Atomic force microscopy (AFM) and transmission electron microscopy (TEM) indicate that single and few layer nanosheets are formed which show crumpling and rolling to form nanoscrolls highlighting the flexibility of these systems. A variety of tri- and hexa-dentate terpyridine (tpy) building blocks have also been used to form monolayers through self-assembly at interfaces through coordination to $\mathrm{M}$ (II) ions ( $\mathrm{Zn}, \mathrm{Co}, \mathrm{Ru}, \mathrm{Pb}, \mathrm{Ni}$ and $\mathrm{Fe}$ ) (Fig. 2d). ${ }^{30,52,75-77}$ These multidentate building blocks provide a high degree of connectivity and robust coordination interactions through their multidentate binding. The often single layered nanosheets which were formed through interfacial assembly can be up to several $\mathrm{cm}$ in lateral dimensions and mechanically strong enough to be spanned over $20 \times 20 \mu \mathrm{m}$ sized holes, however the degree of crystallinity can be low. ${ }^{76} \mathrm{~A}$ structurally related series of MONs have also been synthesised using tri- or tetra-dipyrinato ligands coordinating to tetrahedral zinc ions. ${ }^{78,79}$

\subsection{Diamine/dithiolene based MONs}

A series of MONs assembled from square-planar metal ions and aromatic bis(dithiolenes) have received considerable attention thanks to their remarkable electronic properties.
Benzenehexathiol (BHT) for example was reacted with $\mathrm{d}^{8}$ metal ions including $\mathrm{Ni}(\mathrm{II})^{84}$ and $\mathrm{Pd}(\mathrm{II})^{85}$ to create $2 \mathrm{D}$ planar nanosheets with six-fold symmetry via the formation of the nickel bis(dithiolene) motif (Fig. 2e). These complexes exhibit strong charge delocalization across the three metalladithiolene units through the phenylene linker in mixed-valent states. Expanded versions of these systems have been synthesised using triphenylene hexathiolate with nickel ${ }^{80}$ and cobalt. ${ }^{25}$ The amino analogues of these materials have also been investigated. The interfacial reaction of hexaminobenzene with $\mathrm{Ni}^{2+} / \mathrm{Cu}^{2+} / \mathrm{Co}^{2+}$ acetylacetonate produced flakes several microns wide and approximately $10 \mathrm{~nm}$ thick which were shown to be mildly conducting. Expanded analogues of these layered materials have been formed by reacting hexaminotriphenylene with $\mathrm{Ni}$ and $\mathrm{Cu}$ ions to create semi-conducting frameworks, although individual flakes of these materials have not yet been isolated..$^{\mathbf{2 4 8 6 , 8 7}}$ Mixed amine/thiolene MONs have also been formed with related structures using mixtures of triphenylene hexathiolate and hexaminotriphenylene with $\mathrm{Ni}$ or $\mathrm{Co}^{22}$ and $\mathrm{Ni}(1,3,5-$ triaminobenzene-2,4,6-trithiol). ${ }^{88}$

\subsection{MONs based on other ligand systems}

A number of other MONs have been investigated which are not readily grouped into the classes of MON described above. Notable examples include the earliest example of a single layer thick MON, reported by Zamora and coworkers in 2010. The framework is based on pairs of copper atoms which are bridged by the carboxylate end of two isonicotinato ligands and a bromine ligand and capped by the nitrogen of two further isonicotinato ligands to produce layers (Fig. 2f). ${ }^{81}$ This is an interesting example of a MON with a mixed-valent state and the authors investigate their electrical and magnetic properties. 
Rosseinsky and coworkers investigated a peptide based layered MOF $\left[\mathrm{Zn}(\mathrm{Gly}-\mathrm{Thr})_{2}\right] \cdot \mathrm{CH}_{3} \mathrm{OH}$ which formed single layered nanosheets following exfoliation with ultrasound (Fig. 2g) ${ }^{82}$ Other examples of MONs incorporating multiple coordination motifs within a single framework include a lamellar iron(II)-pyrimidine-2-thiolate coordination polymer $\left[\mathrm{Fe}(\mathrm{Py} 2 \mathrm{th})_{2}\right]_{n}$ (Fig. 2h), ${ }^{\mathbf{8 3}}$ large single layer thick flakes of $[\mathrm{Cu}(\mu-$ $\left.\left.\operatorname{pym}_{2} \mathrm{~S}_{2}\right)(\mu-\mathrm{Cl})\right]_{n}(\text { pyms2 }=\text { dipyrimidindisulfide })^{27}$ and lanthanide based MONs formed by the coordination of 2,2-thiodiacetic acid. ${ }^{89}$ Examples of de-symmetrised MONs with different bonding motifs running in different directions include work by Gao et al. created a zinc based MON with a layered structure consisting of perpendicular chains of carboxylate coordinated ibuprofen and 1,2-bis(4-pyrdiyl)ethane. ${ }^{90}$ Another interesting de-symmetrised case is a two-dimensional coordination polymer consisting of $\mathrm{Cu}_{2} \mathrm{I}_{2}$ chains linked by 2-aminopyrazine. ${ }^{91}$

\section{Synthetic approaches}

There are two distinct approaches to the syntheses of MONs: "bottom-up" methodologies in which the nanosheets are synthesised directly as discreet entities through various methods of arresting the crystallisation process, and "top-down" approaches in which the nanosheets are isolated from bulk layered materials. The coordination bonding present within the layers of MONs is much more dynamic than those of graphene and most other inorganic materials. This opens up opportunities for processing MONs from solution and removes some of the challenges associated with irreversible bond formation. However, it also means milder processes may be required and the structures have the potential to rearrange. Here we discuss various iterations of the two main approaches focussing on examples which have yielded promising nanosheets. A general scheme outlining different approaches is given in Fig. 3 and examples of nanosheets produced by each method are given in Fig. 4 .

\subsection{Arresting crystallisation}

The "bottom-up" synthesis of MONs can be seen as an arrested crystallisation in which growth occurs preferentially in twodimensions. The use of ligands and SBUs with a predisposition to crystallise preferentially in two-dimensions may result in the formation of nanosheets without the need for further modification. For example, Kitagawa and coworkers showed that $\mathrm{Cu}$ (TCPP) MONs of average thickness of $\sim 15 \mathrm{~nm}$, which corresponds to 33 layers, could be synthesised in this manner through a solvothermal synthesis in DMF and EtOH $(3: 1 \mathrm{v} / \mathrm{v})$ (Fig. 4a). ${ }^{47}$

Surfactants have been used to modify crystal habit by binding to the surface of the growing nanosheets, inhibiting growth and preventing stacking of the layers. The surfactant species can also decrease the surface energy and so total energy of the system, which favours the formation of the $2 \mathrm{D}$ morphology. ${ }^{92}$ Zhang and coworkers used polyvinylpyrrolidone (PVP) in the synthesis of $\mathrm{Zn}$ (TCPP). ${ }^{49}$ Addition of PVP to the reactant mixture resulted in MONs of $1.2 \pm 0.4 \mu \mathrm{m} \times 7.6 \pm$ $2.6 \mathrm{~nm}$, corresponding to $8 \pm 3$ layers thick (Fig. 4c). FTIR investigations of PVP interacting with $\mathrm{Zn}^{2+}$ ions showed a strong interaction of the PVP $\mathrm{C}=\mathrm{O}$ group with $\mathrm{Zn}^{2+}$ ions, suggesting that PVP could attach onto the surface of the nanosheet after nucleation, leading to highly anisotropic growth to form MONs. The generality of this method was demonstrated through the additional synthesis of $\mathrm{Cu} / \mathrm{Co} / \mathrm{Cd}(\mathrm{TCPP})$, in which nanosheets of $<10 \mathrm{~nm}$ thickness were obtained for all but Co(TCPP). These MONs have been further used for various applications. ${ }^{\mathbf{5 0 - 5 2 , 5 5}}$ PVP has also been used within the synthesis of $\mathrm{Cu}$ (HBTC) (where $\mathrm{BTC}=1,3,5$-benzene tricarboxylate) in a reaction which occurred at room temperature and pressure to produce nanosheets between $30-55$ nm thick. ${ }^{95}$ The cationic surfactant CTAB has been suggested to adsorb to specific crystal planes. The hydrophobic tail adsorbing to the crystal surface could aid in MON stabilisation and dispersion in particular solvents. This

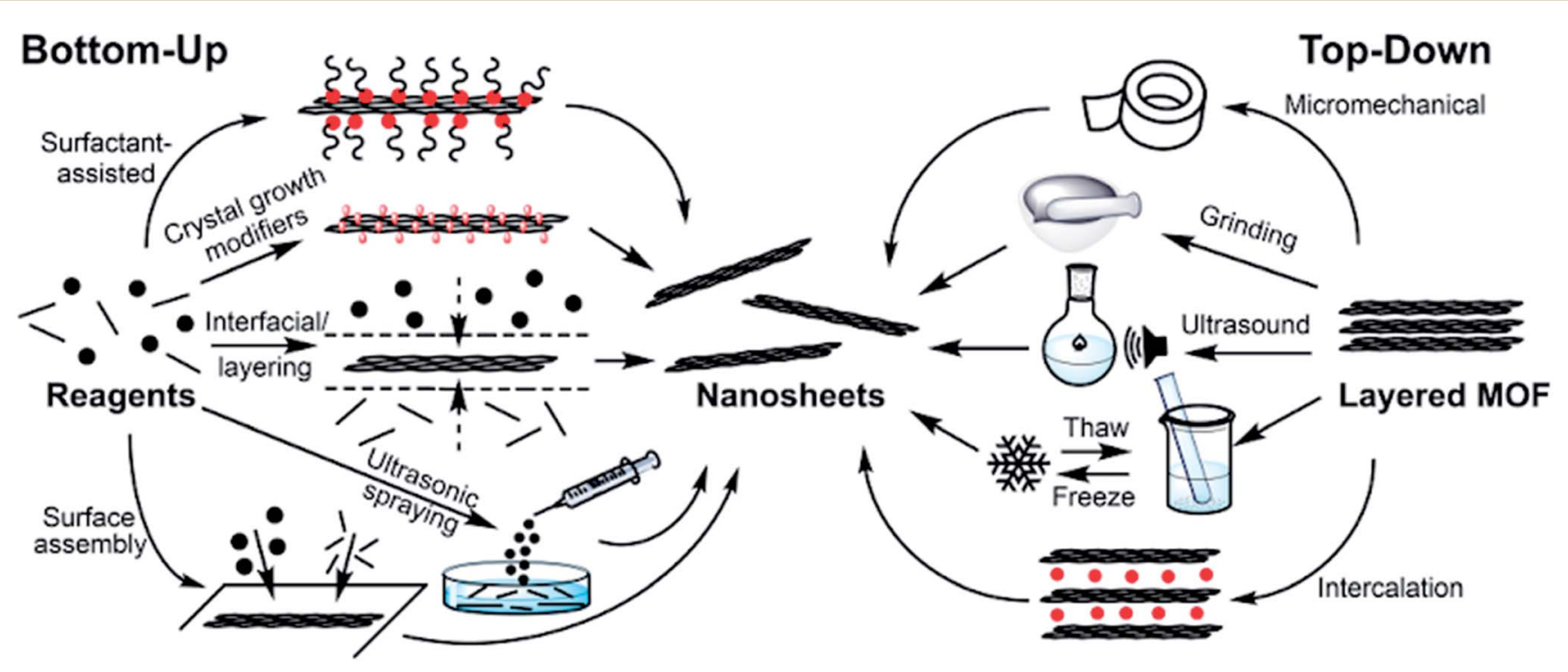

Fig. 3 Scheme showing different bottom-up and top-down methodologies used to produce MONs. 


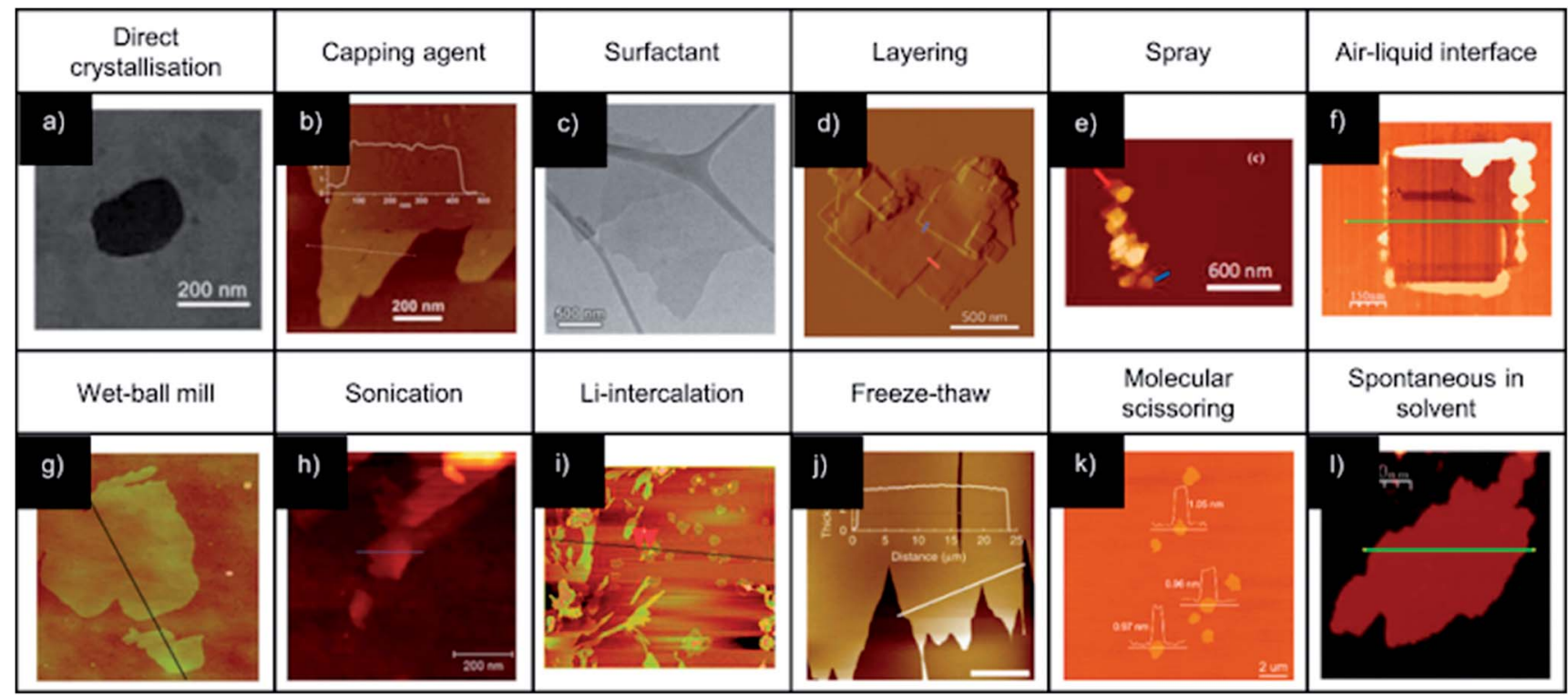

Fig. 4 Example AFM (b, d-l) and TEM $(a, c)$ images illustrating MONs synthesized using various bottom-up (a-f) and top-down ( $\mathrm{g}-\mathrm{l})$ methodologies. Heights of nanosheets across the indicated vectors are approximately: (b) $-3 \mathrm{~nm}$; (d) -6 and $8 \mathrm{~nm}$ (red and blue, respectively), (e) - both $5 \mathrm{~nm},(\mathrm{f})-0.8 \mathrm{~nm},(\mathrm{~g})-1.1 \mathrm{~nm}$, (h) $-1.9 \mathrm{~nm}$, (i) $-2 \mathrm{~nm},(\mathrm{j})-4 \mathrm{~nm}$ and (l) $-2 \mathrm{~nm}$. Image (g) is $3 \mu \mathrm{m}$ square, the indicated vector in (i) is $5 \mu \mathrm{m}$ and the scale bar in (j) is $10 \mu \mathrm{m}$. Images adapted with permission from ref. 47, 93, 49, 36, 37, 30, 68, 43, 89, 40, 53 and 94, for images (a-l) respectively.

approach has been used to directly produce $\mathrm{Zn}(\mathrm{bim})(\mathrm{OAc})$ MONs down to $7 \mathrm{~nm}$ thickness. ${ }^{67,96}$

Small molecule crystal growth modifiers that bind preferentially to a particular facet of a growing crystal can modify the resulting crystal habit. A classic example of this is the addition of pyridine to the synthesis of the pillared MOF $\left[\mathrm{Cu}_{2}(\mathrm{NDC})_{2}\right.$ (DABCO)] (where NDC $=1,4$-naphthalene dicarboxylate and $\mathrm{DABCO}=1,4$-diazabicyclo[2.2.2] octane) which resulted in the formation of large nanosheets up to $500 \mathrm{~nm}^{2}$ as a result of competition with the pillaring ligands. ${ }^{97}$ PVP has been used to similar effect to produce highly anisotropic nanosheets of the pillared MOF Co(TCPP)(BiPY). ${ }^{50}$ It should be noted that these nanosheets are connected in three-dimensions through coordination bonds and therefore do not fulfill our definition of a MON. However, in principle this approach could be used to produce single layers or pillared bilayers which would then have two-dimensional connectivity.

Small molecule addictives can also become incorporated into growing MOFs resulting in the formation of layered structures. Zhao and coworkers exploited acetic acid as a modulator to prevent the formation of interpenetrated networks. This lead to the formation of MONs of 10-20 nm thickness which exhibit stability superior to the analogous 3D MOF (Fig. 4b). ${ }^{93}$ Lin and coworkers found that the 12-connectivity of $\mathrm{Zr}_{6}$ and $\mathrm{Hf}_{6}$ SBUs could be modified by incorporating six formate ions into the SBU to produce nanosheets with thickness of $<4 \mathrm{~nm}$ and lateral dimensions $>10 \mu \mathrm{m}$, with nanosheets observed down to monolayer thickness. ${ }^{23,29,50,61}$

Wang and coworkers demonstrated a "pseudoassembly disassembly" strategy for the formation of $\mathrm{Zr}_{6}$-(NiTCPP) MONs in high yield (85\%) and high dimensional uniformity of around $200 \times 1.5 \mathrm{~nm} .{ }^{59}$ Incorporation of controlled concentrations of small monoacid (formic, acetic, lauric and oleic acid) ligands as a modulator into the reaction mixture lead to layered MOFs, within which the monoacids occupied the interlayer coordination sites of the Zr cluster (confirmed by quantitative NMR and SAXRD) which lead to destabilisation and "disassembly" to MONs. Stacking of produced MONs was observed under TEM with lengthening of the aliphatic chain. Rodenas et al. were able to produce a series of nanosheets of 5-25 $\mathrm{nm}$ thickness and up to $4 \mu \mathrm{m}$ square using a "layered synthesis" method (Fig. $4 \mathrm{~d}$ ). ${ }^{36}$ The ligands and metal ions were dissolved in different ratios of DMF and acetonitrile to produce solutions of different densities which were layered on top of each other, with a buffer layer in between. Slow diffusion of the ligand and metal ions into the buffer layer produced preferential growth in two-dimensions to produce nanosheets, which then sank as a result of gravity into a metal ion deficient layer preventing further growth (Fig. 4e). Inspired by this work, $\mathrm{Fu}$ and coworkers developed a spray technique which used ultrasonic atomisation to spray a solution of metal salt onto a reservoir of the ligand solution. This approach reduces the disturbance caused by contact between the layers, providing a steady interface for anisotropic crystal growth. The nanosheets produced through this method were $<500 \mathrm{~nm}$ and $>5 \mathrm{~nm}$ thick (Fig. 4e).

An alternative method for directing crystal growth into twodimensions is the use of phase interfaces such as air-liquid

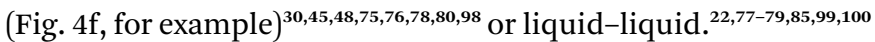
These methods also generally proceed under ambient conditions. Liquid-gas interfacial growth has been demonstrated using Langmuir-Blodgett troughs, in which a solution of ligand in a volatile solvent is applied on top of the aqueous phase containing metal ions. ${ }^{\mathbf{4 6 4 8 , 7 5 , 8 0 , 9 8 , 1 0 1 , 1 0 2}}$ Surface compression can result in large extended nanosheets of potentially infinite size, 
with domains demonstrated up to sub-mm scale, although questions remain about the crystallinity of these large sheets. Additionally, MON films can also be built up by layer-by-layer deposition of these large MONs. More detailed methodology and examples of nanosheets produced in these ways can be found in a recent review by Nishihara and coworkers. ${ }^{29,103,104}$

Additionally, there have been multiple demonstrations since 2003 of the growth of MOF layers at solid-liquid interfaces. These materials have been recently reviewed elsewhere, ${ }^{\mathbf{1 0 5}, \mathbf{1 0 6}}$ and the difficulties associated with removing these materials from the surfaces mean we will not cover these approaches in detail here. Another interesting approach that has been explored is the growth of MOFs on other two-dimensional materials such as graphene oxide, although in most cases the MOFs are not themselves two-dimensional. ${ }^{107}$ Rare examples of the formation of MOF thin films at the vacuum-solid interface by chemical-vapour deposition (CVD) have also been reported, ${ }^{108}$ but again are outside the focus of this review.

\subsection{Exfoliating layered MOFs}

Just as layers of graphite can be separated to form nanosheets of graphene, crystals of layered MOFs can similarly be exfoliated to form MONs. This approach relies on being able to preferentially break apart weak interactions between the layers, without disrupting strong bonding interactions between them.

Abhervé et al. mimicked the famous "Scotch tape" method, originally used to isolate graphene nanosheets, to produce MONs with heights down to $2 \mathrm{~nm}$ from a layered cationic framework. ${ }^{109}$ Exfoliation can also be achieved mechanically, either through grinding by hand or with the use of a ball mill. For example, UiO-67 was exfoliated from the bulk MOF to form sheets of $\sim 10 \mathrm{~nm}$ thickness (Fig. 4g). ${ }^{60}$ This approach produced a relatively broad distribution of particle sizes, in terms of both lateral dimensionality and thickness. Grinding can also cleave chemical bonds, which was utilised by Cliffe et al. to create sheet-like particulates from non-layered MOFs to form nanosheets with a relatively broad distribution of layer thicknesses. ${ }^{60}$ Wet-ball-milling has been additionally demonstrated in a solvent mixture of $\mathrm{MeOH} /{ }^{n} \mathrm{PrOH} .{ }^{68}$ The authors suggest that methanol penetrates into the galleries of the layered MOF, which is facilitated by the ball-milling process, and the ${ }^{n} \mathrm{PrOH}$ acts to stabilize the exfoliated nanosheets through adsorbing onto the surface through the alkane tails, fulfilling a similar role as surfactants previously described.

Liquid exfoliation through sonication has perhaps been the most widely adopted method of MOF exfoliation over the past decade. The energy input provided by the ultrasonicator (typically $20-80 \mathrm{kHz}$, with powers of $80-750 \mathrm{~W}$ ) can overcome the inter-layer interactions, facilitating solvent penetration, and serve as a stimulus for layer separation. As yet, there has not been a universal set of conditions demonstrated to produce the highest quality and quantity of nanosheets. Various authors have used sonication times from 20 minutes to 24 hours, and although exfoliation through sonication has been shown to achieve better results at lower temperature, ${ }^{\mathbf{1 1 0}}$ the temperature of sonication is rarely controlled. Systematically varying centrifugation rates used to collect MONs from suspension can be used to select for different sizes of nanosheet. ${ }^{110}$ The sonication process breaks bonds within the layers as well as between them resulting in smaller crystallite fragments, and subsequently broader particle size distribution and smaller MON size than bottom-up methodology. This is due to the low elastic modulus (3-7 GPa) $)^{27,32}$ of these crystalline materials, compared to other 2D systems such as monolayer graphene and graphene oxide $\left(1000 \pm 100 \mathrm{GPa}\right.$ and $207.6 \pm 23.4 \mathrm{GPa}$ respectively). ${ }^{70,72}$ However, mono- and few-layer nanosheets have been found to be produced through this methodology (Fig. 4h), with lateral dimensions that are comparable to mono/few-layer graphene nanosheets produced from liquid phase graphite exfoliations through sonication, which typically produces nanosheets of below $1 \mu \mathrm{m}$ size. ${ }^{\mathbf{1 1 1}, 112}$

Liquid exfoliation has additionally been achieved by simply stirring, ${ }^{113}$ or shaking a MOF in a solvent (in the presence of surfactant in order to prevent restacking). ${ }^{67}$ Junggeburth et al. note the effect of shaking in differing solvent systems. Their use of a hydrophobic MOF showed decreasing exfoliation in THF > tol $>\mathrm{CHCl}_{3}$, and poor exfoliation observed when using the polar solvents DMF and $\mathrm{H}_{2} \mathrm{O}$. This was suggested to result from an inability of these solvents to efficiently penetrate between the hydrophobic interlayer space. In contrast, Moorthy and coworkers showed that hydrogen bonds between layers of MOF could be overcome by hydrogen-bond-accepting solvents, resulting in spontaneous exfoliation. ${ }^{\mathbf{1 1 4}}$ This shows that the selection of an appropriate solvent system for exfoliation may be of paramount importance. A solvent system that works well for one MOF is not generalizable to others as each MOF has widely varying properties depending on its makeup of metal and ligands. Lower energy-input liquid exfoliation techniques may generally be preferred, as these are less likely to fragment the produced nanosheets.

The intercalation of molecular/ionic species between MOF layers in order to increase interlayer distance and induce exfoliation has been demonstrated for the production of MONs. Lithium-ions were intercalated into various layered frameworks by Wang et al. which were shown to undergo complete exfoliation following sonication in water (Fig. 4i). The forced hydration of the lithium ions is thought to push the layers of MOF apart to such an extent that the interlayer interactions are negated. ${ }^{64,89} \mathrm{~A}$ freeze-thaw method has been demonstrated by Zhou and coworkers, in which a hexane dispersion of MOF was repeatedly frozen in liquid nitrogen and then thawed at elevated temperature with a temperature differential of $256{ }^{\circ} \mathrm{C} .{ }^{40}$ The authors suggest that a shear force was exerted on the MOF crystals as a result of the volumetric change to the hexane upon gas-liquid phase transition. Bilayer thick nanosheets with a broad distribution of lateral dimensions $(10.7 \pm 4.8 \mu \mathrm{m})$ were produced in 95\% yield (Fig. 4j).

The exfoliative techniques discussed so far all rely on the preordered layered 2D structure of MOF, in order to separate layers. However, there is also the potential for chemical modification of the system in order to promote exfoliation. Zhou and coworkers intercalated a pillaring dipyridyl ligand which contained a disulphide bond between layers of a pre-designed 
layered MOF. ${ }^{53}$ This increased the interplanar distance from 9.8 to $22.6 \AA$ A. Scissoring of the ligand through chemical reduction of the disulphide bond resulted in spontaneous exfoliation upon stirring the reaction media, which resulted in single layer nanosheets with lateral dimensions up to several micrometres (Fig. 4k). An element of control upon the produced nanosheet size was additionally demonstrated through varying the reaction conditions for the disulphide scissor. Huang et al. demonstrated an electrochemical exfoliation of a MOF through oxidation of pillaring 2,3-dihydroxybenzene dicarboxylic acid ligands to cyclopenta-2,4-dienone. This allowed for facile removal of the pillars due to weakened coordination ability from both structural strain and electronic differences between the ligands and reported nanosheets with lateral dimensions 100-200 $\mathrm{nm}$ and as little as $2 \mathrm{~nm}$ in height. ${ }^{41}$

In addition, there has been exciting recent work demonstrating solvent induced structural transformations resulting in the formation of nanosheets. Banerjee and coworkers demonstrated a transformative hydrolytic process from metal-organic polyhedra to layered MOF, which resulted in a spontaneous exfoliation to nanosheets of 6-8 layers thick upon addition of larger relative amounts of water. ${ }^{39}$ Similarly, Gallego et al. found that exposure of bulk layered crystals to excess water induced layer separation down to monolayer thickness, without any additional energetic input (Fig. 4l). ${ }^{94}$ This is suggested to be due to incorporation of additional solvent molecules within the cavities present in the starting framework.

\section{Characterisation}

MONs are complex, hierarchical nanomaterials whose structure and properties must be probed using a wide variety of techniques drawn from the solid state, surface, nanomaterials, solution and colloidal characterisation communities. Here we focus on the core-set of methods that have emerged for the characterisation of MONs whilst highlighting innovative use of advanced techniques.

\subsection{Structure and composition}

The prolific success of MOFs has to a large extent been thanks to developments in single-crystal X-ray diffraction (SC-XRD) allowing for atomic resolution of these complex structures. The reduced dimensions of MONs mean the crystals are too small for SC-XRD. The high surface area and often labile structures of metal-organic materials mean that they have a high potential to undergo structural and compositional rearrangements during exfoliation or through subsequent drying or heating of the MONs. ${ }^{36,43}$ However, where the structure of the parent layered MOF or that of a closely related structure is known, comparison or refinement of the X-ray powder diffraction (XRPD) patterns of MONs against the known structure can allow, the structure of the nanosheets to be determined. The powder patterns of MONs measured in reflection or transmission may show systematic loss of peaks corresponding to out of plane reflections in the parent MOF. This may be due to peak broadening resulting from the reduced dimensions of the nanosheet as well as preferred orientation of crystallites. This effect is well illustrated in the work of Gascon and coworkers who were able to assign peaks in the XRPD pattern of their nanosheets to reflections corresponding to the layers which stack along the [201] direction (Fig. 5a). ${ }^{36}$ However, broadening of peaks is not always observed which may indicate reaggregation of nanosheets or larger particles dominating powder patterns. Grazing incidence (GIXRD) can be used to enhance detection of in-plane peaks in thin-films. ${ }^{\mathbf{4 6 , 4 8 , 1 0 1 , 1 0 4}}$

A diverse range of other techniques have also been used to provide additional information about the structure and composition of the MONs. Solid state nuclear magnetic resonance (NMR) spectroscopy, pair distribution function (PDF) data, thermal gravimetric analysis (TGA), infrared spectroscopy and elemental analysis have been widely used to provide evidence of the composition of MONs, as well as NMR analysis of digested samples. ${ }^{23,60,117}$ Surface analysis techniques such as X-ray photo spectroscopy (XPS), X-ray fluorescence (XRF) and Ultraviolet Photoelectron Spectroscopy (UPS) also been used to give information about the elemental composition and oxidation states of the nanosheets (Fig. 5c-f). ${ }^{24,79,89,94,116}$ Density functional theory modelling has also been used alongside experimental data to calculate likely MON structures. ${ }^{60}$

\subsection{Nanoscopic dimensions}

In addition to understanding the molecular structure of MONs, their nanoscopic dimensions must also be probed. AFM represents the gold standard for determining the thickness of nanosheets with angstrom level resolution (Fig. 4 and 6a). In many cases the height of the nanosheets observed match those predicted from the crystal structures. However, the height of multilayer nanosheets may not match those of a single layer a)

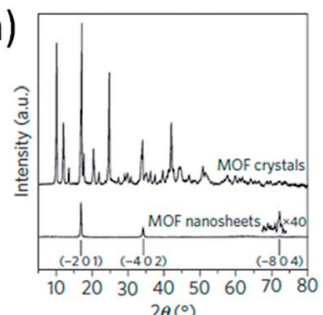

b)

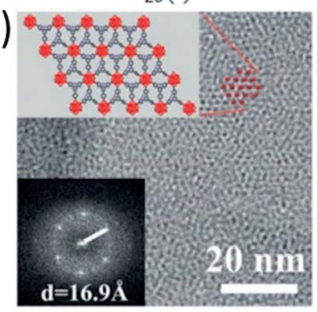

c)
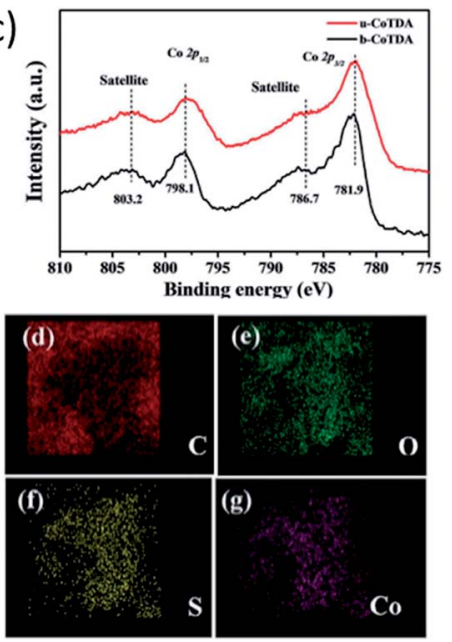

Fig. 5 (a) XRPD patterns comparing nanosheets with parent MOF showing systematic loss of out of plane reflections; (b) STEM-HAADF image of nanosheet showing lattice spacing and corresponding structural model; (c) XPS data providing evidence on elemental composition of a MON; $(d-g)$ EDXS mapping distribution of elements (C, O, S and Co) within a MON. Images reprinted with permission from ref. 36 (a), ref. 115 (b) and ref. 116 (c-g). 

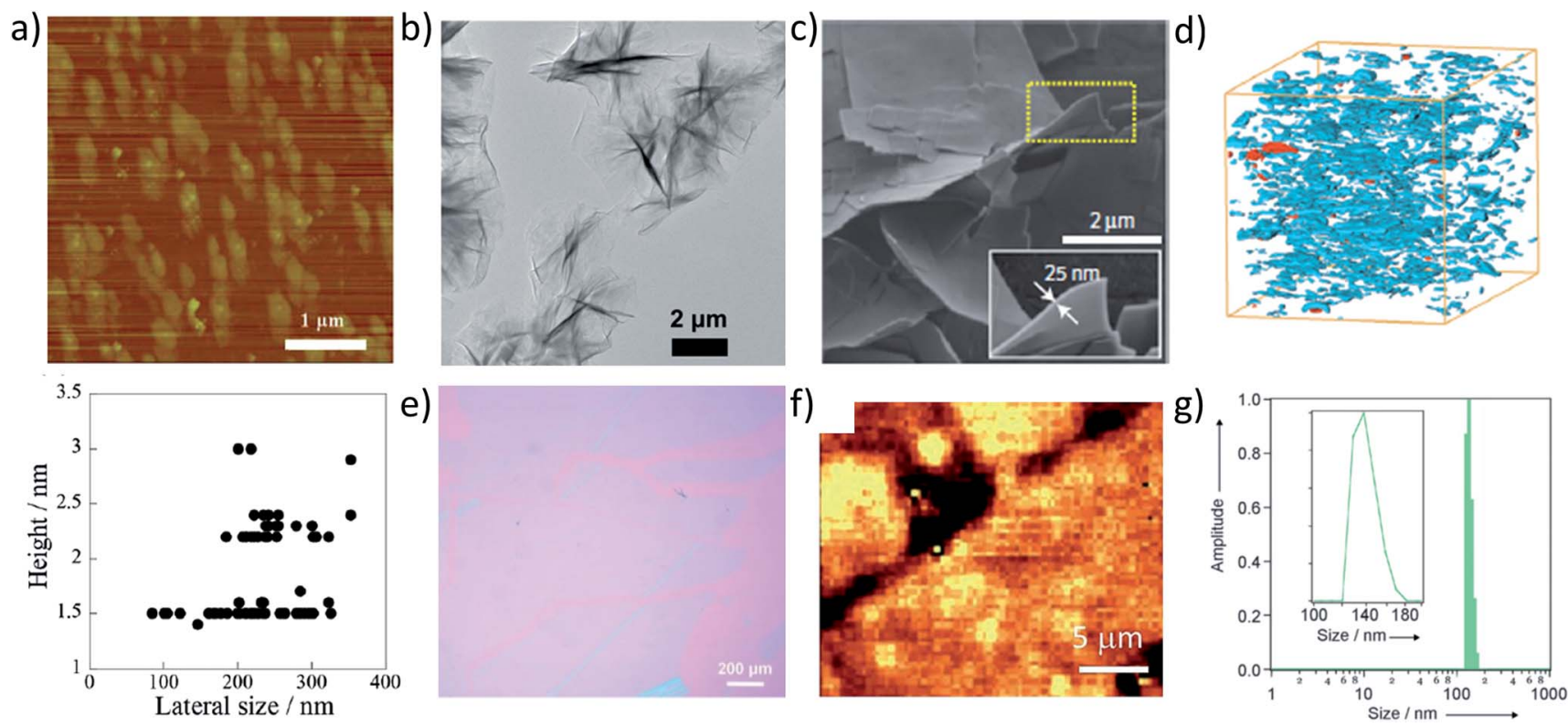

Fig. 6 (a) AFM images of nanosheets and (below) plot of corresponding size profiles; (b) TEM images of crinkled nanosheets; (c) SEM image of nanosheets grown by layering method and associated (d) FEB-SEM of nanosheets dispersed in a polymer membrane; (e) Brewster angle optical microscopy of nanosheets formed at the liquid-gas interface using Langmuir-Blodgett method; (f) Raman microscopy images of MONs; (g) DLS data showing lateral size distribution of nanosheets in suspension. Images reprinted with permission from ref. 74, 56, 36, 30, 27 and 82 respectively.

exactly due to interpenetration of components within layers, as well as instrumental set-up. ${ }^{118}$ The presence of surface water or solvent molecules and counter ions may also lead to nanosheets appearing thicker than expected. ${ }^{68,119}$ MONs have been imaged using a variety of substrates including silica, mica and HOP graphene and different nanosheets will stick to different surfaces to different degrees. Key to imaging the MONs is getting the concentration low enough to see isolated nanosheets. Nanosheets can also aggregate during drying and heating the substrate to speed up drying has been shown to help with imaging other nanosheets. ${ }^{110}$

Scanning electron microscopy (SEM) and TEM can provide high resolution images of the nanosheets, giving information about their dimensions and rigidity. The non-conductive and labile nature of most MONs mean they readily suffer electron damage and charging which can make imaging challenging, although many systems have been successfully imaged with high resolution (Fig. $6 \mathrm{~b}$ and c). A wide variety of advanced electron microscopy techniques have been used to provide additional information about the underlying structure of the MONs. For example, Lin and coworkers used fast Fourier transform and rotation electron diffraction to determine the reciprocal lattice of sub-micron sized nanosheets as well as high resolution TEM and scanning TEM high angle annular darkfield (STEM-HAADF) images to corroborate their structural model (Fig. 5b). ${ }^{23}$ Selected area electron diffraction (SAED) has also been used to confirm unit-cell measurements. ${ }^{29,32}$ Energydispersive X-ray spectroscopy (EDX) and electron energy-loss spectroscopy (EELS) have been used to map the elemental distribution of the nanosheets. ${ }^{59,67,116}$ These powerful techniques provide an important route to understanding the nature and position of defects, functional groups and active sites which are often essential to their performance in a range of applications.

SEM can also achieve good resolution and can be useful for characterising the layers of parent material and getting an overview of the particle size distribution. For example, Rodenas et al. used focused ion beam SEM (FIB-SEM) to image the distribution of MONs within their composite membranes (Fig. 6d) ${ }^{36}$ SEM microscopes can also be coupled to elemental characterisation techniques such as EDX. Brewster angle optical microscopy can be used to image nanosheets at interfaces such as those shown in Fig. $6 \mathrm{~d},{ }^{30}$ as can Raman microscopy (Fig. 6e). ${ }^{27}$

Often only selected images of nanosheets are shown which may not be representative of the bulk sample. Some groups have attempted to quantify the size distribution by imaging a large number of nanosheets and tallying their thickness against their largest lateral dimension, as shown in Fig. 6a taken from the research of Maeda and coworkers. ${ }^{74}$ Although time consuming, this provides a more reliable account of the range of particles observed and must be considered good practice. Dynamic light scattering (DLS) has been used to measure the lateral size distribution of nanosheets. Lin and coworkers observed hydrodynamic diameters of $600-800 \mathrm{~nm}$ which corresponds with wrinkled nanosheets of $\sim 0.5 \times 1 \mu \mathrm{m}$ observed by TEM, ${ }^{61}$ whilst Rosseinsky and coworkers produced a narrow distribution of lateral sizes between 120-180 $\mathrm{nm}$ consistent with those observed by AFM (Fig. 6f). ${ }^{82}$

\subsection{Macroscopic characterisation}

The ability to disperse MONs in solution allows them to be used and processed as suspensions making them available to interact as sensors and catalysts with molecules in solution. The 
presence of Tyndall scattering when a laser is shone through a sample is indicative of the presence of nanosheets in suspension (Fig. 7a). ${ }^{32}$ It should be noted that other shapes of nanoparticle create the same effect and the morphology of the particles in suspension should be evaluated using a suitable imaging technique. The concentration of material that can form a stable suspension is often very low, typically less than $0.1 \mathrm{mg}$ $\mathrm{mL}^{-1}$. However, careful choice of solvent, the presence of surfactants and the incorporation of functional groups can all enhance the concentration of material in suspension. ${ }^{\mathbf{4 3}}$ The concentration of material in suspension can be evaluated by accurately measuring the mass of material left over following careful drying or filtration of a suspension. Alternatively, an estimate can be obtained from UV-vis data by creating a calibration curve from samples of known concentration. ${ }^{43}$ Recently, Moorthy and coworkers were able to compare the degree of exfoliation achieved in different solvents using fluorescence spectroscopy due to quenching of emission upon aggregation or stacking of layers (Fig. 7b). ${ }^{114}$ Elder et al. probed the surface interactions of water and ethanol molecules to $\mathrm{Cu}-\mathrm{BDC}$ nanosheets using temperature-programmed desorption (TPD) and were able to extract kinetic parameters for desorption from edge, pore and external surface sites. ${ }^{38}$

As with MOFs, many of the applications of MONs take advantage of their porous structure and high surface area in the solid state. Gas adsorption measurements have relatively routinely been used to characterise the accessible volumes of MONs (Fig. 7c). ${ }^{\mathbf{6 8 , 1 2 1 , 1 2 2}}$ The Brunauer-Emmett-Teller BET surface area of the nanosheets is typically lower than that of the

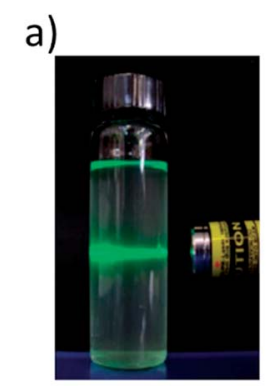

c)

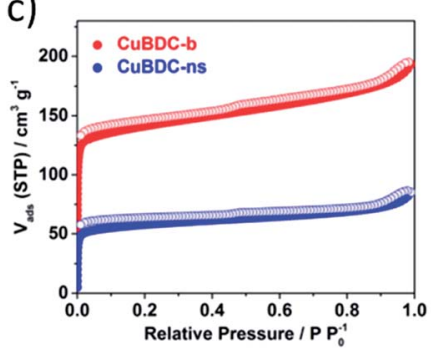

d)

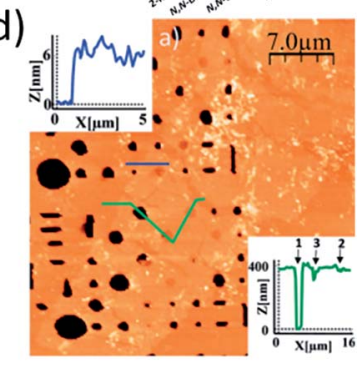

Fig. 7 (a) Photo showing typical Tyndall scattering resulting from a suspension of MONs. (b) Fluorescence emission profiles ( $\lambda_{\text {ex }}=$ $330 \mathrm{~nm}$ ) of $\mathrm{Cd}$ MONs in different solvents. (c) Gas adsorption isotherm comparing $\mathrm{N}_{2}$ uptake for $\mathrm{Cu}(\mathrm{BDC}$ ) bulk MOF (red) and MONs (blue). (d) AFM topographic image of $\mathrm{Cu}$-based nanosheets suspended over micron-sized wells (400 nm depth) within a $\mathrm{Si} / \mathrm{SiO}_{2}$ substrate. Images reprinted with permission from ref. 32, 114, 120 and 27, for images $(a-d)$ respectively. parent bulk porous material due to inefficient packing of the small crystallites, ${ }^{\mathbf{1 2 0}}$ however other properties such as permeability may be significantly enhanced, which is discussed further in Section 5.1.

Understanding the mechanical properties of MONs is also essential for optimising their use in applications such as in membranes for gas separation and in forming composites. The group of Cheetham used a spherical-tip diamond indentor to compare the mechanical properties of different faces of a bulk layered MOF. ${ }^{32}$ They were able to determine the critical resolved shear stress needed for the micromechanical delamination of individual layers to be relatively small at $\leq 0.4$ GPa. AFM can also be used to undertake nanoindentation experiments of nanosheets positioned over wells of different diameters. Gomez-Herrero, Zamora and coworkers calculated the Young's modulus and breaking strength of their frameworks to be $5 \mathrm{GPa}$ and $0.12 \mathrm{~N} \mathrm{~m}^{-1}$ (Fig. 7d). ${ }^{27}$ This is 200 and 150 times lower than the values reported for pristine graphene, but the MONs were still strong enough for free standing sheets to be suspended over micron sized holes.

\section{Applications}

Other metal-organic materials have been developed extensively for a wide variety of applications ranging from 'smart' materials and sensors, to light harvesting, gas storage and drug delivery. MONs share many of the advantages of other metal-organic materials, such as the ease with which their structures can be varied and new properties introduced, allowing them to be tuned for a wide variety of applications. However, their distinct 2D structure, vast external surface area and colloidal nature mean they present a range of opportunities for use in a variety of applications. Here, we seek to illustrate the main bodies of research and recent highlights within the MON literature.

\subsection{Molecular separation}

As with MOFs, MONs can have an open structure creating regular arrays of 1D channels large enough to allow solvent and other molecules to pass through. The tuneable length, sterics and chemistry of the organic linker means that pore size can be controlled to selectively allow certain molecules to pass through, whilst excluding others. The two-dimensional structure of MONs makes them ideal for use in membranes due to the shorter diffusional pathway required as compared to MOFs. Tailoring of the properties of the pore gives opportunities to increase the permeance without a corresponding decrease in specificity. Peng et al. used $1 \mu \mathrm{m} \times 1 \mathrm{~nm}$ ZIF-based MONs as building blocks for a molecular sieving membrane. ${ }^{68,121}$ They achieved a selectivity ratio over 200 for $\mathrm{H}_{2}$ over $\mathrm{CO}_{2}$ (Fig. 8b). Additionally they showed these membranes to be stable over 400 hours of use at room temperature, and for 120 hours at $150{ }^{\circ} \mathrm{C}$ with $4 \mathrm{~mol} \%$ steam. Rodenas et al. found up to an 8 -fold increase in the selectivity of membranes for $\mathrm{CO}_{2}$ over $\mathrm{CH}_{4}$ of upon incorporation of MONs compared with the parent layered MOF. ${ }^{36}$ They attribute this large difference to better packing of the highly anisotropic nanosheets within the membranes, key 

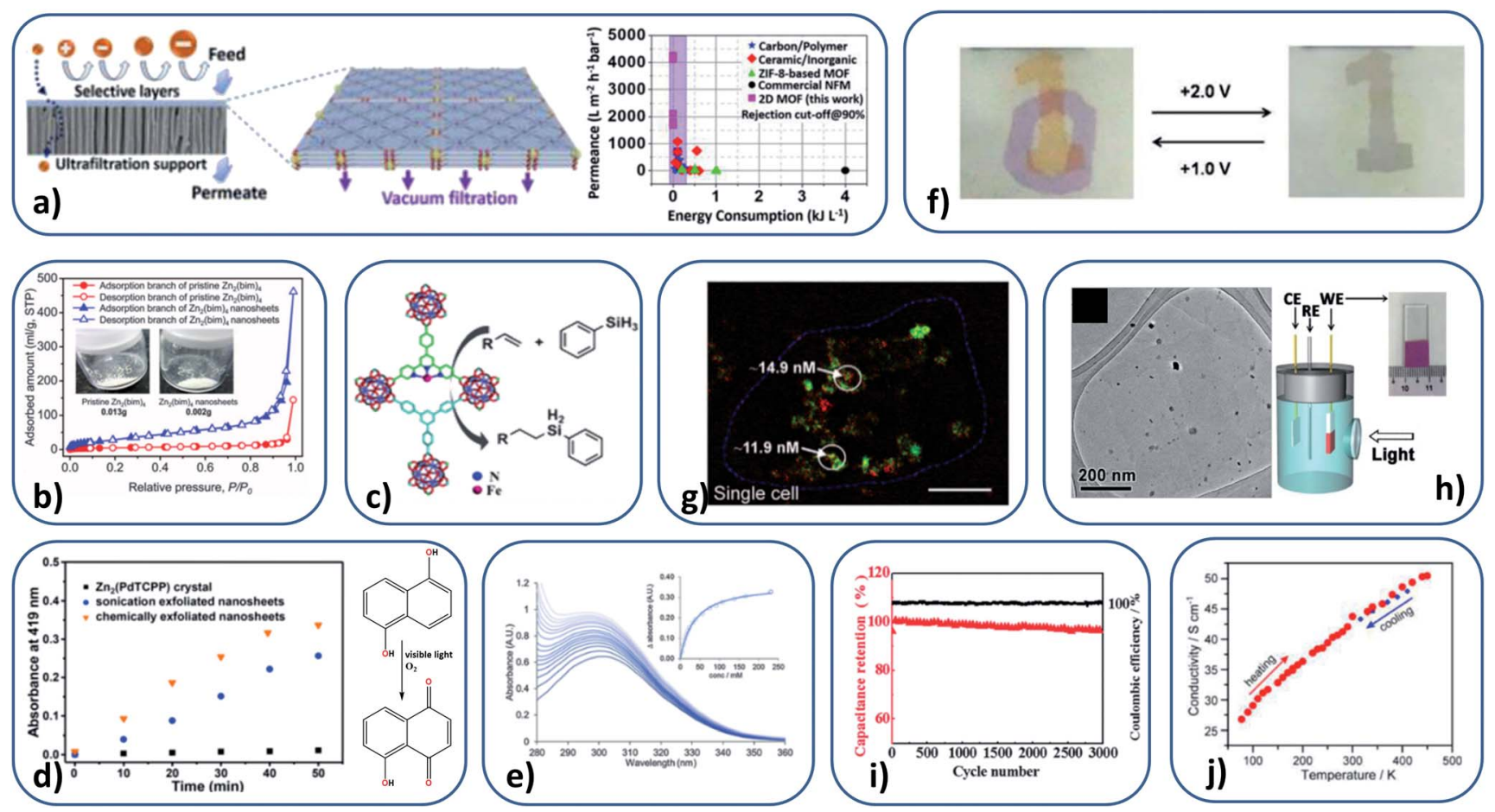

Fig. 8 (a) Schematic illustration of selectively permeable MON composite membrane (left), and permeance as a function of the energy consumption for the 2D M-TCP(Fe)-based membranes, compared with additional advanced membranes. (b) $\mathrm{N}_{2}$ adsorption-desorption isotherms $(77 \mathrm{~K})$ on pristine $\mathrm{Zn}_{2}(\mathrm{bim})_{4}$ and $\mathrm{Zn}_{2}$ (bim) ${ }_{4}$ nanosheets, with inset photographs of material used for analyses. (c) Schematic of catalytic activity of FeTPY MONs. (d) Absorbance of juglone ( $\lambda=419 \mathrm{~nm}$ ) as a function of reaction time with different catalysts (right) and catalytic reaction scheme (left). (e) UV-Vis titration of pyridine into an aqueous suspension of $\mathrm{Cu}-\mathrm{MONs}$, with an inset of absorbance vs. concentration, used to calculate the binding constant. (f) Operation of the dual-electrochromic MON device. (g) Confocal fluorescence image of a single cell, showing two-colour sensing of DNA and small molecules with La-MONs. (h) TEM image of CuS nanoparticle-MON composite (left), and schematic illustration of the used photoelectrochemical cell (right). (i) Charge-discharge profile (black) and specific capacitance (red) at a current density of $2 \mathrm{~A} \mathrm{~g}^{-1}$ of the CoMOF electrode. (j) Variable-temperature van der Pauw conductivity measurement on a $\sim 500 \mathrm{~nm}$ thick MON film on quartz. Images reprinted with permission. Images reprinted with permission from ref. 123, 68, 56, 53, 43, 100, 89, 51, 125 and 24 for images (a-j) respectively.

for the occupation of gas permeation pathways. Further steps in $\mathrm{CO}_{2} / \mathrm{CH}_{4}$ separation have been made by Zhao and coworkers, where a $\mathrm{CO}_{2}$ permeance of $407 \mathrm{GPU}$ was maintained over 100 h. ${ }^{120}$ Interestingly, the same authors have also identified the first reversed thermoswitchable membranes, where $\mathrm{H}_{2}$ permeance decreases at elevated temperatures. ${ }^{40}$ This property is attributed to the flexibility of the $2 \mathrm{D}$ framework leading to blocking of the apertures at elevated temperatures.

Recently, MONs have been used for water purification through high-performance nanofiltration (NF). ${ }^{123}$ Membranes showed permeance about two orders of magnitude higher than a commercial NF membrane, while exhibiting high rejection rates of over $90 \%$ for organic dye molecules with sizes larger than $0.8 \times 1.1 \mathrm{~nm}$ (Fig. 8a). The large body of research on the use of MOFs in gas storage and separation, coupled with the inherent advantages of MON morphology in enhancing the performance of membranes, means this area is likely to see considerable future investment.

\subsection{Catalysis}

The large external surface area of MONs compared to bulk materials makes them ideal candidates as catalysts. They have well-defined structures but can readily be separated from reaction mixtures, combining many of the advantages of homogeneous and heterogeneous catalysts. In MOFs, the majority of active sites are buried within the bulk of the material and reagents must diffuse to the active site. This also limits the size of reagents that may be used and the products that can be formed. The increased on- or near-surface active site presentation of MONs negates these limitations. Various authors have demonstrated that MONs show increased catalytic activity relative to the corresponding 3D MOF, including: hydrosilylation of terminal olefins (Fig. 8c), ${ }^{56} \mathrm{H}_{2} \mathrm{O}_{2}$ reduction (used as biomimetic enzymes for real-time tracking of live cell $\mathrm{H}_{2} \mathrm{O}_{2}$ secretion), ${ }^{52,54}$ and Lewis acid activity for the oxidation of thioanisoles. The cycloaddition of $\mathrm{CO}_{2}$ with epoxides has also been demonstrated. ${ }^{37}$

MONs have also been used as photocatalysts and in electrochemical reactions. Ding et al. demonstrated Zn(Pd-TCPP) MONs perform better in singlet oxygen generation that the unexfoliated MOF, leading to increased rate of photooxidation of 1,5-dihydroxynaphthalene to juglone (Fig. 8d)..$^{53}$ The same photocatalytic reaction has been demonstrated using a $\mathrm{Zr}_{6^{-}}$ cluster-linked (NiTCPP) MON. ${ }^{59}$ Similarly, Zn-(ZnTCPP) MONs were used as a semiconductor photosensitizer along with ZIF-67 as a cocatalyst for the photochemical reduction of $\mathrm{CO}_{2}$ and exhibited enhanced photocatalytic efficiency compared to the 
bulk MOF. ${ }^{55}$ Multiple authors have incorporated MONs into electrodes for use within the electrochemical catalytic splitting of water, for $\mathrm{H}_{2}$ generation..$^{22,25,41,57,80}$ Recent efforts outperform standard $\mathrm{IrO}_{2}$-based catalysts and comparing favourably with state-of-the-art transition-metal-based catalysts. ${ }^{57}$

\subsection{Sensing}

The high external surface area and diverse chemistry of the nanosheets also makes them ideally suited for creating sensors. The binding of analytes to free coordination sites of metal ions, such as the axial position of PW based MONs, can result in changes in the MONs photophysical properties. This allows detection and quantification of binding interactions (Fig. 8d, for example).$^{\mathbf{4 3 , 9 2 , 1 2 4}}$ Similarly, Dincă and coworkers were able to sense ammonia down to $<5 \mathrm{ppm}$ by recording the change in electrical response of the MONs upon substrate coordination. ${ }^{\mathbf{8 6}}$

An alternative approach has been to use MONs to quench the fluorescence of adsorbed, dye-labelled DNA and mRNA, ${ }^{49,64}$ of which detection limits have been found as low as $20 \times$ $10^{-12} \mathrm{M},{ }^{49}$ and $1 \mathrm{pM}$ (after hybridisation chain reaction amplification). ${ }^{64}$ Similarly, background flourescence quenching enabled a signal-to-noise ratio increase of $7.5 \times$ that when using 2D graphene oxide, and enabled a detection limit down to 0.9 pM (0.3 pg mL $\left.{ }^{-1}\right)$ of chloramphenical, a model antibiotic, ${ }^{126}$ and luminescent quenching of nanosheet photoluminescence has been used to detect $\mathrm{Fe}^{3+}$ to a detection limit of $0.45 \mu \mathrm{M}$, amongst additional metal ions, ${ }^{127}$ and $0.054 \mu \mathrm{M}$ (compared with $0.11 \mu \mathrm{M}$ for bulk MOF). ${ }^{128}$

He et al. have developed MONs within a biosensor for the protein MUC1, with a detection limit of $0.12 \mathrm{ng} \mathrm{mL}{ }^{-1} \cdot{ }^{129}$ The cancer marker protein was detected from human serum, which points towards MON application within medicinal biosensing and diagnoses. Wang et al. have additionally demonstrated a lanthanide-based MON, useful as a two-colour sensing platform for intracellular DNA and small molecules (Fig. 8g). ${ }^{89}$ For further information on the preparation of MONs for chemical and biosensing and examples within this area, see Yang et al.'s recent review. ${ }^{\mathbf{1 3 0}}$

\subsection{Electronics}

Perhaps the greatest excitement surrounding graphene has been the possibility of harnessing its electronic properties to create a new generation of ultrathin electronic devices. Graphene can potentially be combined with other 2D materials with insulating or semiconducting properties in order to form ultrathin electronic devices. In 2013, Nishihara and coworkers demonstrated a planer $\pi$-conjugated MON which was shown to be semiconducting, opening up research into MONs within molecular electronics. ${ }^{99}$ Since then, multiple authors have found MONs to be semiconducting, ${ }^{24,84,86,88,99,131,132}$ with Dincă and coworkers setting a record in 2014 for conductivity of metal-organic materials, at $40 \mathrm{~S} \mathrm{~cm}^{-1}$ (Fig. 8i). ${ }^{24}$ Zeng and coworkers have suggested from theoretical calculations that it may be possible to tune semiconducting MONs to metals through alteration of the metal ions used. ${ }^{87}$ Additional work fabricating MONs into electrochemical devices has suggested
MONs may be useful within devices such as colour displays and electronic paper, due to their rapid electrochemical response rate (Fig. 8f). ${ }^{\mathbf{1 0 0 , 1 3 2}}$

Recent work has been directed towards the incorporation of MONs into supercapacitor electrodes..$^{\mathbf{5 0 , 1 2 5 , 1 3 3}}$ The groups of Jia and Wei have utilised MONs as electrode materials. Jia and coworkers found an $80.2 \%$ retention rate after 500 chargedischarge cycles, ${ }^{\mathbf{1 3 3}}$ and more recently the group of Wei set records for both capacitance $\left(2564 \mathrm{~F} \mathrm{~g}^{-1}\right)$ and retention $(95.8 \%$ after 3000 cycles) for MO materials (Fig. 8i). ${ }^{125}$

\subsection{Photofunctional nanomaterials}

As with other new materials, researchers seek novel, inventive uses for them to further the body of research and excitement surrounding them. Lin and coworkers have recently demonstrated that fluorescence of few-layer MONs can be used in white light emitting diodes (WLEDs), which exhibit a switching speed of at least three times as fast as current commercial WLEDs, due to the shorter fluorescence lifetime of MONs. This makes them useful for visible-light communications, used within wireless information transmission applications. ${ }^{23}$ The group of Nishihara have demonstrated an avenue into development of MONs into photofunctional devices. ${ }^{77}$ They found photoluminescent colour could be modified through alteration of both the metal ions and ligands used, as well as almost quantitative exciton transmission to bound guest dyes. This opens up possibilities of further development for novel photofunctional nanomaterials.

\subsection{Other applications}

MONs have been used as a surface in order to grow in situ $\mathrm{M}-\mathrm{S}$ nanoparticles $(\mathrm{M}=\mathrm{Cu}, \mathrm{Cd}, \mathrm{Co})$, using the metal sites presented on the nanosheet surface to stimulate growth (Fig. 8h). ${ }^{51}$ This led to the CuS-nanosheet composite performing with notably increased photocurrent within a photoelectrochemical cell, compared to the nanosheet alone. This is due to the improved exciton separation and charge carrier transport from the CuS nanoparticles. Zhang and coworkers suggest these types of composite MON materials may have applications within solar cells and photochromism, for example.

Liu et al. have utilised MONs as a clean-background matrix for MALDI-TOF mass spectrometry analysis of small biomolecules. ${ }^{69}$ Biomarkers are normally below the scope of this technique as interference from conventional matrices normally limits the size of molecules that can be seen to $\sim 700 \mathrm{Da}$. The superior background provided by the nanosheet composite allowed detection of glutamic acid (147 Da) amongst other small molecules and biomarkers. The same group also demonstrate MON inhibition of enzyme activity, allowing activity modulation. ${ }^{113}$

\section{Conclusions and outlook}

The extensive body of research on MOFs has provided an important starting point for identifying promising layered compounds and SBUs for the construction of MONs. A diverse 
range of MOF SBUs and ligands readily provide the strong, directional interactions within two-dimensions required to form MONs and innovation has already occurred in adapting well known SBUs to give two-dimensional connectivity. Other key MOF concepts such as reticular substitution and postsynthetic modification are also already beginning to be used in MONs with great effect. However, the design principles behind creating the porous, three-dimensional structures which have been the focus of the MOF community may be quite distinct from those required to create effective two-dimensional MONs. For example, in MOFs neutral frameworks have generally been favoured as counterions tend to occupy pore space. However, in MONs counterions can sit above or below the nanosheets and aid formation of nanosheets through solvation. Structural rearrangements may occur in nanosheets which do not occur in bulk structures due to the high surface area and MONs may form higher order structures such as rolling into "nanoscrolls." We anticipate that as research into MONs develops, new SBUs and design features not found in MOF chemistry will emerge to address the distinct requirements and opportunities faced in forming nanosheets from metal-organic building blocks.

Intensive research over the last decade into the synthesis of two-dimensional materials and the exfoliation of layered materials into nanosheets similarly provides an important starting point for research into MONs. The use of ultrasound, solvation, intercalants, surfactants and crystal-growth modifiers all have parallels in the formation of nanosheets from other materials. There has been considerable innovation over the last year in new 'softer forms' of exfoliation and new ways to direct crystal growth, but it is not yet clear how broadly applicable these approaches will be. As with other nanosheets, the preferred method of synthesis is likely to depend on the properties of nanosheets required. In general, bottom-up methods have tended to produce larger nanosheets with narrower size distributions whilst top-down exfoliations have tended to give smaller, thinner sheets. However, the diversity of MON chemistry means it is difficult to generalise and compare different studies, and in most cases the synthesis or exfoliation will not have been optimised. More detailed and systematic studies are therefore required to understand the effect of different parameters on the dimensions of nanosheets produced to allow more targeted synthesis.

A diverse range of solid, solution, colloidal and surface analysis techniques must be combined to provide insights into different levels of their structure. The complex molecular structure of MONs, their relatively fragile and non-conducting composition and the spread of sizes and thicknesses of nanosheets produced makes them particularly challenging to characterise. Greater understanding of the mechanical, optoelectronic and porosity of nanosheets and how this compares with the parent, layered materials will be important for identifying new applications. There is also little general acceptance of what constitutes a "nanosheet" in terms of the number of layers, size distribution and crystallinity of the materials formed. Setting the bar too high at this early stage has the potential to stifle innovation. Setting it too low will dilute the field with compounds without the potential to form freestanding, crystalline, single-layered materials.

The success of MONs as a distinct class of nanomaterials is likely to rest on their utility. The billions of dollars invested by industry and funding councils in other two-dimensional and metal-organic materials demonstrates both the potential demand for these type of materials and the challenges in bringing them to market. The chemical diversity, modular structure and tunability of MONs offers obvious advantages over simple inorganic nanosheets and the inherent reversibility of coordination bonds and unique properties of metal ions offers distinct opportunities compared to covalent-organic framework (COF) nanosheets. The high external surface area and tuneable structure of MONs make them obvious candidates for a wide range of sensing, separation and catalytic applications and there are already promising examples of MONs outperforming bulk MOFs when used as membranes for gasseparation and as catalysts. The tuneable electronic, optical and magnetic properties of MONs also potentially allow them to be used as layers in diverse range of devices including displays, solar-cells and batteries. The wide range of inexpensive and abundant ligands and metal ions that can be used to construct MONs and the ability to synthesise them from solution and process them as suspensions offers further environmental and economic advantages.

In conclusion, MONs represent a rich new class of materials requiring distinct approaches in their design, synthesis and characterisation and offering a novel combination of properties that can be harnessed for a wide range of applications. This is an emerging field whose boundaries, techniques, rules and potential are only just beginning to be explored. Here we have tried to highlight both the breadth of different approaches that have so far been taken and draw out common threads between the different studies. We hope that this review will further stimulate this exciting area of research and look forward to contributing further to its development.

\section{Conflicts of interest}

There are no conflicts to declare.

\section{Acknowledgements}

JAF would like to thank the Ramsey Memorial Fellowship and DJA the Engineering, Physical Sciences Research Council for funding (EP/K503149/1).

\section{References}

1 T. Torres, Chem. Soc. Rev., 2017, 46, 4385-4386.

2 J. Wang, F. Ma and M. Sun, RSC Adv., 2017, 7, 16801-16822.

3 Y. Zhou, M. Zhang, Z. Guo, L. Miao, S.-T. Han, Z. Wang, X. Zhang, H. Zhang and Z. Peng, Mater. Horiz., 2017, 4, 997-1019.

4 H. T. Tan, W. P. Sun, L. B. Wang and Q. Y. Yan, ChemNanoMat, 2016, 2, 562-577.

5 H. Yin and Z. Tang, Chem. Soc. Rev., 2016, 45, 4873-4891. 
6 R. Lv, J. A. Robinson, R. E. Schaak, D. Sun, Y. F. Sun, T. E. Mallouk and M. Terrones, Accounts Chem. Res., 2015, 48, 56-64.

7 L. Xu and J. Sun, Adv. Energy Mater., 2016, 6, 1600441.

8 M. Pan, Z. W. Wei, Y. W. Xu and C. Y. Su, Prog. Chem., 2017, 29, 47-74.

9 J. Y. Zhang and C. Y. Su, Coord. Chem. Rev., 2013, 257, 13731408.

10 R. Gaillac, P. Pullumbi, K. A. Beyer, K. W. Chapman, D. A. Keen, T. D. Bennett and F. X. Coudert, Nat. Mater., 2017, 16, 1149-1154.

11 J. L. Serrano and T. Sierra, Coord. Chem. Rev., 2003, 242, 7385.

12 A. Winter and U. S. Schubert, Chem. Soc. Rev., 2016, 45, 5311-5357.

13 P. Z. Moghadam, A. Li, S. B. Wiggin, A. Tao, A. G. P. Maloney, P. A. Wood, S. C. Ward and D. FairenJimenez, Chem. Mater., 2017, 29, 2618-2625.

14 B. Li, H. M. Wen, W. Zhou and B. Chen, J. Phys. Chem. Lett., 2014, 5, 3468-3479.

15 E. M. Dias and C. Petit, J. Mater. Chem. A, 2015, 3, 2248422506.

16 M. Ranocchiari and J. A. van Bokhoven, Phys. Chem. Chem. Phys., 2011, 13, 6388-6396.

17 X. L. Jiang, H. H. Wu, S. J. Chang, R. Si, S. Miao, W. X. Huang, Y. H. Li, G. X. Wang and X. H. Bao, J. Mater. Chem. A, 2017, 5, 19371-19377.

18 L. E. Kreno, K. Leong, O. K. Farha, M. Allendorf, R. P. Van Duyne and J. T. Hupp, Chem. Rev., 2012, 112, 1105-1125.

19 H. S. Wang, Coord. Chem. Rev., 2017, 349, 139-155.

20 I. Stassen, N. Burtch, A. Talin, P. Falcaro, M. Allendorf and R. Ameloot, Chem. Soc. Rev., 2017, 46, 3185-3241.

21 M. Zhao, Q. Lu, Q. Ma and H. Zhang, Small Methods, 2016, 1, 1600030.

22 R. Dong, Z. Zheng, D. C. Tranca, J. Zhang, N. Chandrasekhar, S. Liu, X. Zhuang, G. Seifert and X. Feng, Chem.-Eur. J., 2017, 23, 2255-2260.

23 X. Hu, Z. Wang, B. Lin, C. Zhang, L. Cao, T. Wang, J. Zhang, C. Wang and W. Lin, Chem.-Eur. J., 2017, 23, 8390-8394.

24 D. Sheberla, L. Sun, M. A. Blood-Forsythe, S. Er, C. R. Wade, C. K. Brozek, A. Aspuru-Guzik and M. Dincă, J. Am. Chem. Soc., 2014, 136, 8859-8862.

25 A. J. Clough, J. W. Yoo, M. H. Mecklenburg and S. C. Marinescu, J. Am. Chem. Soc., 2015, 137, 118-121.

26 C. Kutzscher, A. Gelbert, S. Ehrling, C. Schenk, I. Senkovska and S. Kaskel, Dalton Trans., 2017, 46, 16480-16484.

27 C. Hermosa, B. R. Horrocks, J. I. Martinez, F. Liscio, J. Gomez-Herrero and F. Zamora, Chem. Sci., 2015, 6, 2553-2558.

28 D. Rodriguez-San-Miguel, P. Amo-Ochoa and F. Zamora, Chem. Commun., 2016, 52, 4113-4127.

29 R. Sakamoto, K. Takada, T. Pal, H. Maeda, T. Kambe and H. Nishihara, Chem. Commun., 2017, 53, 5781-5801.

30 T. Bauer, Z. Zheng, A. Renn, R. Enning, A. Stemmer, J. Sakamoto and A. D. Schlüter, Angew. Chem., Int. Ed., 2011, 50, 7879-7884.

31 G. Z. Zhu and Q. Sun, Comp. Mater. Sci., 2016, 112, 492-502.
32 J.-C. Tan, P. J. Saines, E. G. Bithell and A. K. Cheetham, ACS Nano, 2012, 6, 615-621.

33 C. Lee, X. Wei, J. W. Kysar and J. Hone, Science, 2008, 321, 385-388.

34 A. K. Cheetham, C. N. R. Rao and R. K. Feller, Chem. Commun., 2006, 46, 4780-4795.

35 P. Z. Li, Y. Maeda and Q. Xu, Chem. Commun., 2011, 47, 8436-8438.

36 T. Rodenas, I. Luz, G. Prieto, B. Seoane, H. Miro, A. Corma, F. Kapteijn, F. X. Llabrés, i. Xamena and J. Gascon, Nat. Mater., 2015, 14, 48-55.

37 Y.-N. Li, S. Wang, Y. Zhou, X.-J. Bai, G.-S. Song, X.-Y. Zhao, T.-Q. Wang, X. Qi, X.-M. Zhang and Y. Fu, Langmuir, 2017, 33, 1060-1065.

38 A. C. Elder, A. B. Aleksandrov, S. Nair and T. M. Orlando, Langmuir, 2017, 33, 10153-10160.

39 B. Garai, A. Mallick, A. Das, R. Mukherjee and R. Banerjee, Chem.-Eur. J., 2017, 23, 7361-7366.

40 X. Wang, C. Chi, K. Zhang, Y. Qian, K. M. Gupta, Z. Kang, J. Jiang and D. Zhao, Nat. Commun., 2017, 8, 14460.

41 J. Huang, Y. Li, R. K. Huang, C. T. He, L. Gong, Q. Hu, L. Wang, Y. T. Xu, X. Y. Tian, S. Y. Liu, Z. M. Ye, F. Wang, D. D. Zhou, W. X. Zhang and J. P. Zhang, Angew. Chem., Int. Ed., 2018, 57, 4632-4636.

42 Y. Hou, L. Liu, S. Qiu, X. Zhou, Z. Gui and Y. Hu, ACS Appl. Mater. Interfaces, 2018, 10, 8274-8286.

43 J. A. Foster, S. Henke, A. Schneemann, R. A. Fischer and A. K. Cheetham, Chem. Commun., 2016, 52, 10474-10477.

44 R. Makiura, S. Motoyama, Y. Umemura, H. Yamanaka, O. Sakata and H. Kitagawa, Nat. Mater., 2010, 9, 565-571.

45 R. Makiura, K. Tsuchiyama and O. Sakata, CrystEngComm, 2011, 13, 5538-5541.

46 S. Motoyama, R. Makiura, O. Sakata and H. Kitagawa, J. Am. Chem. Soc., 2011, 133, 5640-5643.

47 G. Xu, T. Yamada, K. Otsubo, S. Sakaida and H. Kitagawa, J. Am. Chem. Soc., 2012, 134, 16524-16527.

48 R. Makiura, R. Usui, Y. Sakai, A. Nomoto, A. Ogawa, O. Sakata and A. Fujiwara, ChemPlusChem, 2014, 79, 1352-1360.

49 M. Zhao, Y. Wang, Q. Ma, Y. Huang, X. Zhang, J. Ping, Z. Zhang, Q. Lu, Y. Yu, H. Xu, Y. Zhao and H. Zhang, Adv. Mater., 2015, 27, 7372-7378.

50 F. Cao, M. Zhao, Y. Yu, B. Chen, Y. Huang, J. Yang, X. Cao, Q. Lu, X. Zhang, Z. Zhang, C. Tan and H. Zhang, J. Am. Chem. Soc., 2016, 138, 6924-6927.

51 Q. Lu, M. T. Zhao, J. Z. Chen, B. Chen, C. Tan, X. Zhang, Y. Huang, J. Yang, F. Cao, Y. Yu, J. Ping, Z. Zhang, X.-J. Wu and H. Zhang, Small, 2016, 12, 4669-4674.

52 Y. Wang, M. Zhao, J. Ping, B. Chen, X. Cao, Y. Huang, C. Tan, Q. Ma, S. Wu, Y. Yu, Q. Lu, J. Chen, W. Zhao, Y. Ying and H. Zhang, Adv. Mater., 2016, 28, 4149-4155.

53 Y. Ding, Y. P. Chen, X. Zhang, L. Chen, Z. Dong, H. L. Jiang, H. Xu and H. C. Zhou, J. Am. Chem. Soc., 2017, 139, 91369139.

54 Y. Huang, M. T. Zhao, S. K. Han, Z. C. Lai, J. Yang, C. L. Tan, Q. L. Ma, Q. P. Lu, J. Z. Chen, X. Zhang, Z. C. Zhang, B. Li, B. Chen, Y. Zong and H. Zhang, Adv. Mater., 2017, 29, 1700102 . 
55 L. Ye, Y. Gao, S. Cao, H. Chen, Y. Yao, J. Hou and L. Sun, Appl. Catal., B, 2018, 227, 54-60.

56 L. Cao, Z. Lin, F. Peng, W. Wang, R. Huang, C. Wang, J. Yan, J. Liang, Z. Zhang, T. Zhang, L. Long, J. Sun and W. Lin, Angew. Chem., Int. Ed., 2016, 128, 5046-5050.

57 L. Zhao, B. Dong, S. Li, L. Zhou, L. Lai, Z. Wang, S. Zhao, M. Han, K. Gao, M. Lu, X. Xie, B. Chen, Z. Liu, X. Wang, H. Zhang, H. Li, J. Liu, H. Zhang, X. Huang and W. Huang, ACS Nano, 2017, 11, 5800-5807.

58 Y. Wang, L. Li, L. Yan, X. Gu, P. Dai, D. Liu, J. G. Bell, G. Zhao, X. Zhao and K. M. Thomas, Chem. Mater., 2018, 30, 3048-3059.

59 T. He, B. Ni, S. Zhang, Y. Gong, H. Wang, L. Gu, J. Zhuang, W. Hu and X. Wang, Small, 2018, 14(16), 1703929.

60 M. J. Cliffe, E. Castillo-Martinez, Y. Wu, J. Lee, A. C. Forse, F. C. N. Firth, P. Z. Moghadam, D. Fairen-Jimenez, M. W. Gaultois, J. A. Hill, O. V. Magdysyuk, B. Slater, A. L. Goodwin and C. P. Grey, J. Am. Chem. Soc., 2017, 139, 5397-5404.

61 L. Cao, Z. Lin, W. Shi, Z. Wang, C. Zhang, X. Hu, C. Wang and W. Lin, J. Am. Chem. Soc., 2017, 139, 7020-7029.

62 P. J. Saines, J.-C. Tan, H. H. M. Yeung, P. T. Barton and A. K. Cheetham, Dalton Trans., 2012, 41, 8585-8593.

63 P. J. Saines, M. Steinmann, J.-C. Tan, H. H. M. Yeung, W. Li, P. T. Barton and A. K. Cheetham, Inorg. Chem., 2012, 51, 11198-11209.

64 W.-J. Song, Talanta, 2017, 170, 74-80.

65 T. Araki, A. Kondo and K. Maeda, Chem. Commun., 2013, 49, 552-554.

66 R. Chen, J. Yao, Q. Gu, S. Smeets, C. Baerlocher, H. Gu, D. Zhu, W. Morris, O. M. Yaghi and H. Wang, Chem. Commun., 2013, 49, 9500-9502.

67 S. C. Junggeburth, L. Diehl, S. Werner, V. Duppel, W. Sigle and B. V. Lotsch, J. Am. Chem. Soc., 2013, 135, 6157-6164.

68 Y. Peng, Y. Li, Y. Ban, H. Jin, W. Jiao, X. Liu and W. Yang, Science, 2014, 346, 1356-1359.

69 H. L. Liu, Y. J. Chang, T. Fan and Z. Y. Gu, Chem. Commun., 2016, 52, 12984-12987.

70 Y. Lo and D. Y. Kang, J. Mater. Chem. A, 2016, 4, 4172-4179.

71 Q. Liu, Z. X. Low, Y. Feng, S. Leong, Z. X. Zhong, J. F. Yao, K. Hapgood and H. T. Wang, Microporous Mesoporous Mater., 2014, 194, 1-7.

72 H. Jiang, S. L. Xue, Y. F. Liu, W. H. Xing and R. Z. Chen, Microporous Mesoporous Mater., 2017, 243, 16-21.

73 L. Huang, X. P. Zhang, Y. J. Han, Q. Q. Wang, Y. X. Fang and S. J. Dong, J. Mater. Chem. A, 2017, 5, 18610-18617.

74 A. Kondo, C. C. Tiew, F. Moriguchi and K. Maeda, Dalton Trans., 2013, 42, 15267-15270.

75 Z. Zheng, C. S. Ruiz-Vargas, T. Bauer, A. Rossi, P. Payamyar, A. Schuetz, A. Stemmer, J. Sakamoto and A. D. Schlueter, Macromol. Rapid Commun., 2013, 34, 1670-1680.

76 Z. Zheng, L. Opilik, F. Schiffmann, W. Liu, G. Bergamini, P. Ceroni, L.-T. Lee, A. Schuetz, J. Sakamoto, R. Zenobi, J. VandeVondele and A. D. Schlueter, J. Am. Chem. Soc., 2014, 136, 6103-6110.

77 T. Tsukamoto, K. Takada, R. Sakamoto, R. Matsuoka, R. Toyoda, H. Maeda, T. Yagi, M. Nishikawa, N. Shinjo,
S. Amano, T. Iokawa, N. Ishibashi, T. Oi, K. Kanayama, R. Kinugawa, Y. Koda, T. Komura, S. Nakajima, R. Fukuyama, N. Fuse, M. Mizui, M. Miyasaki, Y. Yamashita, K. Yamada, W. Zhang, R. Han, W. Liu, T. Tsubomura and H. Nishihara, J. Am. Chem. Soc., 2017, 139, 5359-5366.

78 R. Sakamoto, K. Hoshiko, Q. Liu, T. Yagi, T. Nagayama, S. Kusaka, M. Tsuchiya, Y. Kitagawa, W.-Y. Wong and H. Nishihara, Nat. Commun., 2015, 6, 6713.

79 R. Sakamoto, T. Yagi, K. Hoshiko, S. Kusaka, R. Matsuoka, H. Maeda, Z. Liu, Q. Liu, W.-Y. Wong and H. Nishihara, Angew. Chem., Int. Ed., 2017, 56, 3526-3530.

80 R. Dong, M. Pfeffermann, H. Liang, Z. Zheng, X. Zhu, J. Zhang and X. Feng, Angew. Chem., Int. Ed., 2015, 54, 12058-12063.

81 P. Amo-Ochoa, L. Welte, R. Gonzalez-Prieto, P. J. Sanz Miguel, C. J. Gomez-Garcia, E. Mateo-Marti, S. Delgado, J. Gomez-Herrero and F. Zamora, Chem. Commun., 2010, 46, 3262-3264.

82 C. Marti-Gastaldo, J. E. Warren, K. C. Stylianou, N. L. O. Flack and M. J. Rosseinsky, Angew. Chem., Int. Ed., 2012, 51, 11044-11048.

83 P. J. Beldon, S. Tominaka, P. Singh, T. S. Dasgupta, E. G. Bithell and A. K. Cheetham, Chem. Commun., 2014, 50, 3955-3957.

84 K. Hoshiko, T. Kambe, R. Sakamoto, K. Takada and H. Nishihara, Chem. Lett., 2014, 43, 252-253.

85 T. Pal, T. Kambe, T. Kusamoto, M. L. Foo, R. Matsuoka, R. Sakamoto and H. Nishihara, ChemPlusChem, 2015, 80, 1255-1258.

86 M. G. Campbell, D. Sheberla, S. F. Liu, T. M. Swager and M. Dinca, Angew. Chem., Int. Ed., 2015, 54, 4349-4352.

87 S. Chen, J. Dai and X. C. Zeng, Phys. Chem. Chem. Phys., 2015, 17, 5954-5958.

88 X. Sun, K.-H. Wu, R. Sakamoto, T. Kusamoto, H. Maeda and H. Nishihara, Chem. Lett., 2017, 46, 1072-1075.

89 H.-S. Wang, J. Li, J.-Y. Li, K. Wang, Y. Ding and X.-H. Xia, NPG Asia Mater., 2017, 9, e354.

90 X. Gao, R. Cui, M. Zhang and Z. Liu, Mater. Lett., 2017, 197, 217-220.

91 J. Conesa-Egea, J. Gallardo-Martinez, S. Delgado, J. I. Martinez, J. Gonzalez-Platas, V. Fernandez-Moreira, U. R. Rodriguez-Mendoza, P. Ocon, F. Zamora and P. Amo-Ochoa, Small, 2017, 13, 1700965.

92 Y. P. Yuan, W. Wang, L. G. Qiu, F. M. Peng, X. Jiang, A. J. Xie, Y. H. Shen, X. Y. Tian and L. D. Zhang, Mater. Chem. Phys., 2011, 131, 358-361.

93 Z. Hu, E. M. Mahdi, Y. Peng, Y. Qian, B. Zhang, N. Yan, D. Yuan, J.-C. Tan and D. Zhao, J. Mater. Chem. A, 2017, 5, 8954-8963.

94 A. Gallego, C. Hermosa, O. Castillo, I. Berlanga, C. J. GomezGarcia, E. Mateo-Marti, J. I. Martinez, F. Flores, C. GomezNavarro, J. Gomez-Herrero, S. Delgado and F. Zamora, Adv. Mater., 2013, 25, 2141-2146.

95 G. Zhan and H. C. Zeng, Adv. Funct. Mater., 2016, 26, 3268-3281.

96 F. Xue, P. Kumar, W. Xu, K. A. Mkhoyan and M. Tsapatsis, Chem. Mater., 2017, 30, 69-73. 
97 M.-H. Pham, G.-T. Vuong, F.-G. Fontaine and T.-O. Do, Cryst. Growth Des., 2012, 12, 3091-3095.

98 R. Makiura and O. Konovalov, Dalton Trans., 2013, 42, 15931-15936.

99 T. Kambe, R. Sakamoto, K. Hoshiko, K. Takada, M. Miyachi, J.-H. Ryu, S. Sasaki, J. Kim, K. Nakazato, M. Takata and H. Nishihara, J. Am. Chem. Soc., 2013, 135, 2462-2465.

100 K. Takada, R. Sakamoto, S.-T. Yi, S. Katagiri, T. Kambe and H. Nishihara, J. Am. Chem. Soc., 2015, 137, 4681-4689.

101 R. Makiura and O. Konovalov, Sci. Rep., 2013, 3, 2506.

102 G. Xu, K. Otsubo, T. Yamada, S. Sakaida and H. Kitagawa, J. Am. Chem. Soc., 2013, 135, 7438-7441.

103 R. Sakamoto, K. Takada, X. Sun, T. Pal, T. Tsukamoto, E. J. H. Phua, A. Rapakousiou, K. Hoshiko and H. Nishihara, Coord. Chem. Rev., 2016, 320, 118-128.

104 H. Maeda, R. Sakamoto and H. Nishihara, Langmuir, 2016, 32, 2527-2538.

105 J. X. Liu and C. Woll, Chem. Soc. Rev., 2017, 46, 5730-5770. 106 V. Rubio-Gimenez, M. Galbiati, J. Castells-Gil, N. AlmoraBarrios, J. Navarro-Sanchez, G. Escorcia-Ariza, M. Mattera, T. Arnold, J. Rawle, S. Tatay, E. Coronado and C. MartiGastaldo, Adv. Mater., 2018, 30, 8.

107 S. Li, K. Yang, C. Tan, X. Huang, W. Huang and H. Zhang, Chem. Commun., 2016, 52, 1555-1562.

108 K. Sakata, S. Kashiyama, G. Matsuo, S. Uemura, N. Kimizuka and M. Kunitake, ChemNanoMat, 2015, 1, 259-263.

109 A. Abhervé, S. Manas-Valero, M. Clemente-Leon and E. Coronado, Chem. Sci., 2015, 6, 4665-4673.

110 C. Backes, T. M. Higgins, A. Kelly, C. Boland, A. Harvey, D. Hanlon and J. N. Coleman, Chem. Mater., 2017, 29, 243-255.

111 A. O'Neill, U. Khan, P. N. Nirmalraj, J. Boland and J. N. Coleman, J. Phys. Chem. C, 2011, 115, 5422-5428.

112 A. Ciesielski and P. Samori, Chem. Soc. Rev., 2014, 43, 381-398.

113 M. Xu, S. Yuan, X. Y. Chen, Y. J. Chang, G. Day, Z. Y. Gu and H. C. Zhou, J. Am. Chem. Soc., 2017, 139, 8312-8319.

114 P. Chandrasekhar, A. Mukhopadhyay, G. Savitha and J. N. Moorthy, J. Mater. Chem. A, 2017, 5, 5402-5412.

115 W. Shi, L. Cao, H. Zhang, X. Zhou, B. An, Z. Lin, R. Dai, J. Li, C. Wang and W. Lin, Angew. Chem., Int. Ed., 2017, 56, 97049709.
116 Y. Ning, X. Lou, C. Li, X. Hu and B. Hu, Chem.-Eur. J., 2017, 23, 15984-15990.

117 H. S. Quah, L. T. Ng, B. Donnadieu, G. K. Tan and J. J. Vittal, Inorg. Chem., 2016, 55, 10851-10854.

118 C. J. Shearer, A. D. Slattery, A. J. Stapleton, J. G. Shapter and C. T. Gibson, Nanotechnology, 2016, 27, 125704.

119 P. Nemes-Incze, Z. Osváth, K. Kamarás and L. P. Biró, Carbon, 2008, 46, 1435-1442.

120 Y. D. Cheng, X. R. Wang, C. K. Jia, Y. X. Wang, L. Z. Zhai, Q. Wang and D. Zhao, J. Membr. Sci., 2017, 539, 213-223.

121 Y. Peng, Y. Li, Y. Ban and W. Yang, Angew. Chem., Int. Ed., 2017, 56, 9757-9761.

122 Z. Kang, Y. Peng, Z. Hu, Y. Qian, C. Chi, L. Y. Yeo, L. Tee and D. Zhao, J. Mater. Chem. A, 2015, 3, 20801-20810.

123 H. X. Ang and L. Hong, ACS Appl. Mater. Interfaces, 2017, 9, 28079-28088.

124 Z.-Q. Li, L.-G. Qiu, W. Wang, T. Xu, Y. Wu and X. Jiang, Inorg. Chem. Commun., 2008, 11, 1375-1377.

125 J. Yang, Z. Ma, W. Gao and M. Wei, Chem.-Eur. J., 2017, 23, 631-636.

126 Q. Yang, L. Zhou, Y.-X. Wu, K. Zhang, Y. Cao, Y. Zhou, D. Wu, F. Hu and N. Gan, Anal. Chim. Acta, 2018, 1020, 1-8.

127 H. Xu, J. Gao, X. Qian, J. Wang, H. He, Y. Cui, Y. Yang, Z. Wang and G. Qian, J. Mater. Chem. A, 2016, 4, 1090010905.

128 L. J. Han, D. Zheng, S. G. Chen, H. G. Zheng and J. Ma, Small, 2018, 14(17), e1703873.

129 L. He, F. Duan, Y. Song, C. Guo, H. Zhao, J.-Y. Tian, Z. Zhang, C.-S. Liu, X. Zhang, P. Wang, M. Du and S.-M. Fang, 2D Mater., 2017, 4, 025098.

130 T. Yang, Y. N. Cui, H. Y. Chen and W. H. Li, Acta Chim. Sin., 2017, 75, 339-350.

131 S. Benmansour, A. Abherve, P. Gomez-Claramunt, C. VallesGarcia and C. J. Gomez-Garcia, ACS Appl. Mater. Interfaces, 2017, 9, 26210-26218.

132 N. Lahiri, N. Lotfizadeh, R. Tsuchikawa, V. V. Deshpande and J. Louie, J. Am. Chem. Soc., 2017, 139, 19-22.

133 Z. P. Sun, L. Hui, W. S. Ran, Y. Lu and D. Z. Jia, New J. Chem., 2016, 40, 1100-1103. 